\title{
Overlap-Driven Splitting of Triplet Pairs in Singlet Fission
}

\author{
Elliot J. Taffet, ${ }^{1,2}$ David Beljonne ${ }^{3}$ and Gregory D. Scholes ${ }^{* 4}$ \\ 1 Department of Chemistry and the PULSE Institute, Stanford \\ University, Stanford, California 94305, USA \\ ${ }^{2}$ SLAC National ACCelerator Laboratory, Menlo Park, California \\ 94025, USA \\ 3 Department of Chemistry, University of Mons, 7000 Mons, Belgium \\ ${ }^{4}$ Department of Chemistry, Princeton University, Princeton, New \\ Jersey 08544, United States
}

\section{Analysis of the Optimized DMRG Wavefunctions}

We identify the properties of the computationally resolved wavefunctions and demonstrate the correspondence with theoretical representations of TT states. The main identifier of ${ }^{1} \mathrm{TT}$ electronic structure is the unpaired-electron-number metric, ${ }^{1}$ which operates on the one-particle reduced density matrix of the state to determine the extent to which the DMRG wavefunction resembles the tetraradicaloid (four-unpaired-spin) configuration of pure TT:

$$
N_{\text {odd }}=2\left(\operatorname{Tr}[\gamma]-\operatorname{Tr}\left[\gamma \gamma^{+}\right]\right)=2\left(N-\operatorname{Tr}\left[\gamma \gamma^{+}\right]\right)
$$

Where $\mathrm{N}_{\text {odd }}$ is the unpaired-electron number and $\gamma$ is the one-particle reduced density matrix.

We compute $\mathrm{N}_{\text {odd }}$ for all adiabatic ${ }^{1} \mathrm{TT}$ states computed at the DMRG(4,4)-SCF level of theory. The results, illustrated in Figure S1, reveal the direct correspondence between 
configurational impurity - the deviation of $\mathrm{N}_{\text {odd }}$ from 4-and exchange splitting $(J)$ caused by this configurational mixing.

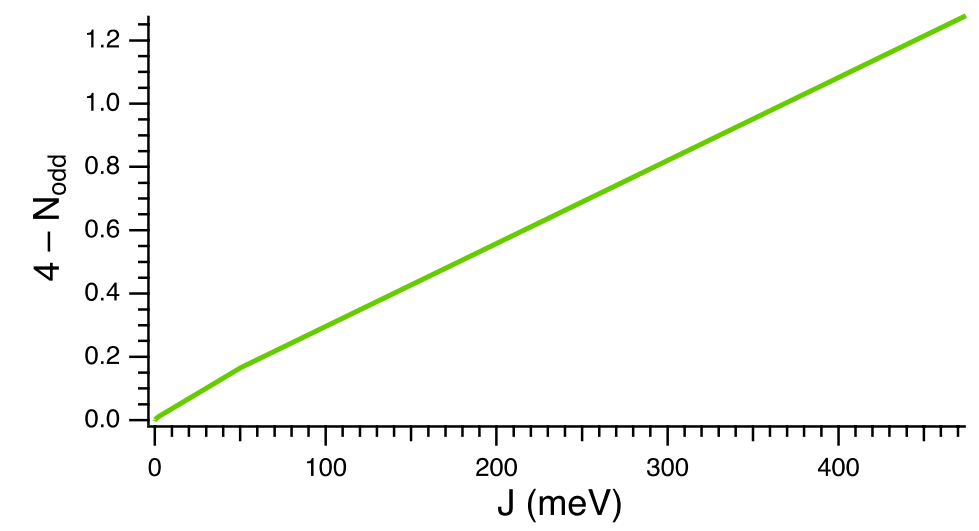

Figure S1. Direct correspondence between the purity of the ${ }^{1} \mathrm{TT}$ state (as quantified by $4-\mathrm{N}_{\text {odd }}$ ) and the exchange splitting $(J)$ between the TT spin-states.

Table S1 displays the results on the individual systems, including a residual-error metric based on deviation from the theoretical exchange-split ladder of TT states $\left({ }^{1} \mathrm{TT}={ }^{3} \mathrm{TT}-J={ }^{5} \mathrm{TT}-3 J\right)$. Table S1. System-Specific Unpaired-Electron-Number $\left(\mathrm{N}_{\text {odd }}\right)$ and Exchange-Splitting $(J)$ Values.

\begin{tabular}{|l|l|l|}
\hline System & $4-\mathrm{N}_{\text {odd }}$ & $J(\mathrm{meV})$ \\
\hline TIPS-Tetracene Dimer 1 & 0.00105 & $0.067 \pm 9.19 \times 10^{-5}$ \\
\hline TIPS-Tetracene Dimer 2 & $8.24 \times 10^{-9}$ & $0.002 \pm 1.62 \times 10^{-4}$ \\
\hline TIPS-Tetracene Dimer 3 & $2.78 \times 10^{-6}$ & $0.0003 \pm 1.24 \times 10^{-4}$ \\
\hline TIPS-Tetracene Dimer 4 & $2.30 \times 10^{-13}$ & 0 \\
\hline TIPS-Tetracene Dimer 5 & $1.24 \times 10^{-7}$ & 0 \\
\hline Bipentacene & 0.010 & $2.14 \pm 0.061$ \\
\hline BTDO1 & 0.166 & $50.4 \pm 8.03$ \\
\hline Neurosporene & 0.861 & $658 \pm 586$ \\
\hline
\end{tabular}


Critically, the exchange-splitting values — even those on the sub- $\mu \mathrm{eV}$ scale - are distinguishable through this computational approach as signal above the numerical noise defined as the residual error in the computed exchange-split ladder.

In addition to analysis of the one-particle reduced density matrix, we may analyze the two- and one-particle transition density matrices for additional physical insight into the resemblance of the computational TT intermediates to the pure T...T product of singlet fission. In the two-particle transition-density-matrix picture with an active space of four orbitals numbered 1-4, the signature TT double-excitation takes the double-charge-transfer form (23|14). That is, the TT double-excitation, in the face of alternating frontier orbitals on each monomer, is the product of an excitation from monomer 1 to monomer 2 together with an excitation from monomer 2 to monomer 1 . We find that those computational TT states with the largestmagnitude (23|14) two-particle transition-density matrix elements correspondingly have the smallest transition dipole moments (as ascertained from the one-particle transition density matrix), which we attribute to greater configurational purity with respect to the signature doublecharge-transfer excitation (Table S2).

Table S2. System-Specific Two- and One-Particle Transition-Density-Matrix Properties.

\begin{tabular}{|l|l|l|}
\hline System & $(23 \mid 14)$ Matrix Element & Transition Dipole Moment \\
\hline TIPS-Tetracene Dimer 1 & -1.48 & 0.00186 Debye \\
\hline TIPS-Tetracene Dimer 2 & 1.64 & $1.69 \times 10^{-6}$ Debye \\
\hline TIPS-Tetracene Dimer 3 & 1.63 & $8.84 \times 10^{-6}$ Debye \\
\hline TIPS-Tetracene Dimer 4 & 1.64 & $5.45 \times 10^{-11}$ Debye \\
\hline TIPS-Tetracene Dimer 5 & -1.64 & $2.88 \times 10^{-6}$ Debye \\
\hline Bipentacene & -0.765 & 0.00101 Debye \\
\hline
\end{tabular}




\begin{tabular}{|l|l|l|}
\hline BTDO1 & -0.808 & $2.91 \times 10^{-4}$ Debye \\
\hline Neurosporene & -0.616 & 0.660 Debye \\
\hline
\end{tabular}

We also may turn to energetic properties of the underlying triplet states contributing to the TT excitation by examining Davydov splitting between the computed $T_{1}$ and $T_{2}$ states, as well as the energetic difference between ${ }^{1} \mathrm{TT}$ and $\left(\mathrm{T}_{1}+\mathrm{T}_{2}\right)$. In the nonoverlapping regime between molecular units, the energies of $\mathrm{T}_{1}$ and $\mathrm{T}_{2}$, as well as $\left(\mathrm{T}_{1}+\mathrm{T}_{2}\right),{ }^{1} \mathrm{TT},{ }^{3} \mathrm{TT}$ and ${ }^{5} \mathrm{TT}$, are equivalent. ${ }^{2}$ Energetic deviations, however, arise due to excitonic effects between the triplets in the overlapping regime, ${ }^{2}$ thereby splitting the formally monomeric triplets due to excitondelocalization and increasing the $\left(\mathrm{T}_{1}+\mathrm{T}_{2}\right)$ energy relative to ${ }^{1} \mathrm{TT}$ due to overlap-driven configuration-interaction. The energetic relationships $\left(T_{2}-T_{1}\right)$ (Davydov splitting) and $\left(T_{1}+\right.$ $\mathrm{T}_{2}$ ) - ${ }^{1} \mathrm{TT}$ for slip-displaced geometries of TIPS-Tetracene Dimer 1 are enumerated in Table S3. Table S3. System-Specific Deviations from Idealized Energetic Relationships (Exciton-Effects).

\begin{tabular}{|l|l|l|}
\hline TIPS-Tetracene Dimer 1 & $\left(\mathrm{~T}_{2}-\mathrm{T}_{1}\right)$ Relative Energy & Ditto $\left(\mathrm{T}_{1}+\mathrm{T}_{2}\right)-{ }^{1} \mathrm{TT}$ \\
\hline Crystal & $7.40 \mathrm{meV}$ & $0.407 \mathrm{meV}$ \\
\hline Slip-Displacement $0.1 \AA$ & $6.43 \mathrm{meV}$ & $0.256 \mathrm{meV}$ \\
\hline Slip-Displacement $0.2 \AA$ & $5.76 \mathrm{meV}$ & $0.208 \mathrm{meV}$ \\
\hline Slip-Displacement $0.3 \AA$ & $5.09 \mathrm{meV}$ & $0.174 \mathrm{meV}$ \\
\hline Slip-Displacement $0.4 \AA$ & $4.43 \mathrm{meV}$ & $0.150 \mathrm{meV}$ \\
\hline Slip-Displacement $0.5 \AA$ & $3.81 \mathrm{meV}$ & $0.134 \mathrm{meV}$ \\
\hline Slip-Displacement $0.6 \AA$ & $3.05 \mathrm{meV}$ & $0.124 \mathrm{meV}$ \\
\hline Slip-Displacement $0.7 \AA$ & $2.43 \mathrm{meV}$ & $0.117 \mathrm{meV}$ \\
\hline Slip-Displacement $0.8 \AA$ & $1.92 \mathrm{meV}$ & $0.111 \mathrm{meV}$ \\
\hline
\end{tabular}




\begin{tabular}{|l|l|l|}
\hline Slip-Displacement $0.9 \AA$ & $1.54 \mathrm{meV}$ & $0.106 \mathrm{meV}$ \\
\hline Slip-Displacement $1.0 \AA$ & $1.21 \mathrm{meV}$ & $0.102 \mathrm{meV}$ \\
\hline Slip-Displacement $1.1 \AA$ & $0.992 \mathrm{meV}$ & $0.098 \mathrm{meV}$ \\
\hline Slip-Displacement $1.2 \AA$ & $0.871 \mathrm{meV}$ & $0.095 \mathrm{meV}$ \\
\hline Slip-Displacement $1.3 \AA$ & $0.784 \mathrm{meV}$ & $0.091 \mathrm{meV}$ \\
\hline Slip-Displacement $1.4 \AA$ & $0.763 \mathrm{meV}$ & $0.087 \mathrm{meV}$ \\
\hline
\end{tabular}

We thus observe monotonicity in the decay of the Davydov splitting $\left(T_{2}-T_{1}\right)$ and the excitonic splitting $\left(\mathrm{T}_{1}+\mathrm{T}_{2}\right)-{ }^{1} \mathrm{TT}$ as the monomers of TIPS-Tetracene Dimer 1 are separated. Therefore, we may revisit the curves plotted in Figure 1 of the main text within the context of these exciton-splitting quantities listed in Table S3 as presented below in Figure S2.

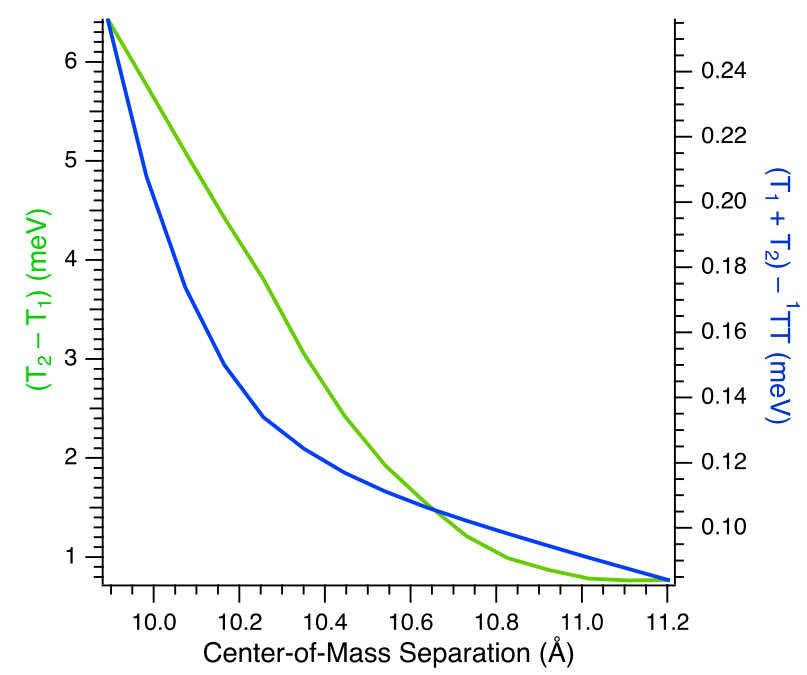

Figure S2. The monotonic decay of each exciton-splitting quantity with respect to increasing center-of-mass separation length of TIPS-tetracene dimer 1 . The left axis corresponds to Davydov splitting between the triplet states, while the right axis corresponds to the interactioninduced relative stabilization of ${ }^{1} \mathrm{TT}$. 
We now turn to an excitonic picture of interaction matrices between diabatic (configurationally pure) states to explain the Davydov splitting (an "overlap energy" effect) and the ${ }^{1} \mathrm{TT}$ energetic stabilization relative to the sum of Davydov-split triplet energies (an "interaction energy" effect). First, let us turn to the triplet excitonic picture of mixing between two locally excited triplet $\left(\mathrm{T}_{\mathrm{LE}}\right)$ monomeric states and two charge-resonance triplet $\left(\mathrm{T}_{\mathrm{CR}}\right)$ dimeric states. Let us assume that the diabatic $\mathrm{T}_{\mathrm{LE}}$ and $\mathrm{T}_{\mathrm{CR}}$ energies are diagonally corrected within the interaction matrix by explicit orbital nonorthogonality between the monomers - an overlap correction that stabilizes the diabats. We may couple these overlap-corrected diabats in the following matrix:

$$
\begin{array}{ccccc}
T_{L E} & \left(h_{A} h_{B} \mid l_{A} l_{B}\right) & \left(l_{A} \mid l_{B}\right) & \left(h_{B} \mid h_{A}\right) \\
\left(h_{A} h_{B} \mid l_{A} l_{B}\right) & T_{L E} & \left(h_{A} \mid h_{B}\right) & \left(l_{B} \mid l_{A}\right) \\
\left(l_{A} \mid l_{B}\right) & \left(h_{A} \mid h_{B}\right) & T_{C R} & \left(h_{A} h_{B} \mid l_{A} l_{B}\right) \\
\left(h_{B} \mid h_{A}\right) & \left(l_{B} \mid l_{A}\right) & \left(h_{A} h_{B} \mid l_{A} l_{B}\right) & T_{C R}
\end{array}
$$

The diabats listed in the diagonal elements of the above symmetric interaction matrix take on the following configurations for monomers $\mathrm{A}$ and $\mathrm{B}$ of the dimer from top left to bottom right: $\mathrm{A}^{\mathrm{t}} \mathrm{B}^{0}, \mathrm{~A}^{0} \mathrm{~B}^{\mathrm{t}},\left(\mathrm{A}^{+} \mathrm{B}^{-}\right)^{\mathrm{t}},\left(\mathrm{A}^{-} \mathrm{B}^{+}\right)^{\mathrm{t}}\left({ }^{\mathrm{t}}\right.$ denotes a triplet state, while ${ }^{0}$ denotes a ground state). The $\mathrm{h}$ and 1 frontier orbitals (as defined in Table 1 of the main text) are indexed according to their monomeric origin (monomer A or monomer B). The off-diagonal matrix elements-that is, the electronic couplings between the diabatic configurations - are labeled according to their leading electron-repulsion-integral terms.

We may observe that two-electron Dexter exchange terms couple the $\mathrm{T}_{\mathrm{LE}}$ states, thereby explaining the splitting between the triplets quantified in Table $\mathrm{S} 3$. The sum of these split $\mathrm{T}_{\mathrm{LE}}$ energies, in the absence of mixing with $\mathrm{T}_{\mathrm{CR}}$ configurations, corresponds to the noninteracting ${ }^{5} \mathrm{TT}$ energy that is stabilized solely by the orbital overlap stabilizing the $\mathrm{T}_{\mathrm{LE}}$ diabats. 
Nonetheless, the mixing between $\mathrm{T}_{\mathrm{LE}}$ and $\mathrm{T}_{\mathrm{CR}}$ diabatic configurations, dominated by stronger one-electron coupling terms as opposed to the two-electron coupling between $\mathrm{T}_{\mathrm{LE}}$ diabats, may profoundly lower the triplet adiabatic energies. This effect is most pronounced in the carotenoid neurosporene that is examined in the main text, where the overlap-driven mixing between the $\mathrm{T}_{\mathrm{LE}}$ diabats on the two half-chain molecular units and the delocalized $\mathrm{T}_{\mathrm{CR}}$ diabats strongly stabilizes the symmetric Davydov $T_{1}$ component relative to the $T_{L E}$ energy. As a result, twice the $T_{1}$ energy lies far below the first ${ }^{5} \mathrm{TT}$ state, corresponding to twice the $\mathrm{T}_{\mathrm{LE}}$ diabatic energy in the above interaction matrix, within neurosporene. The $T_{2}$ adiabatic energy, however, lies close to that of $T_{\mathrm{LE}}$, largely because of the reduced effective coupling to $\mathrm{T}_{\mathrm{CR}}$ and the smaller mixinginduced stabilization of the anti-symmetric Davydov triplet component.

Therefore, as presented in Figure $2 \mathrm{~b}$ of the main text, twice the $\mathrm{T}_{2}$ energy corresponds to the energy of the lowest lying ${ }^{5} \mathrm{TT}$ state, which itself corresponds to twice the diagonally corrected $T_{L E}$ energy. In sum, the Davydov splitting between the triplets $\left(T_{2}-T_{1}\right)$ is a manifestation of Dexter exchange coupling, or the overlap energy that stabilizes ${ }^{5} \mathrm{TT}$ relative to $\mathrm{T} \ldots \mathrm{T}$ that itself energetically corresponds to twice the diagonally uncorrected $\mathrm{T}_{\mathrm{LE}}$ energy (that is, the energy of the molecular triplet without an energetic correction due to orbital nonorthogonality).

On the other hand, the interaction energy is the additional configuration-interaction between ${ }^{1} \mathrm{TT}$ and the $\mathrm{S}_{\mathrm{LE}} / \mathrm{S}_{\mathrm{CR}}$ diabats (pairs of spin-singlet locally excited and charge-resonance configurations), which will stabilize the coupled triplet-triplet pair relative to the adiabatic energies of the triplets that comprise it, as well as the noninteracting ${ }^{5} \mathrm{TT}$ energy. When ${ }^{1} \mathrm{TT}$ is $2^{1} \mathrm{~A}_{\mathrm{g}}$ (the lowest lying ${ }^{1} \mathrm{TT}$ state that is energetically related to ${ }^{3} \mathrm{~B}_{\mathrm{u}}+{ }^{3} \mathrm{~B}_{\mathrm{u}}$ ), the energetic ordering will be ${ }^{1} \mathrm{TT}_{\mathrm{AB}}<\mathrm{T}_{1}+\mathrm{T}_{2}<{ }^{5} \mathrm{TT}_{\mathrm{AB}}$. This ordering is due to the weaker ${ }^{3} \mathrm{~T}_{\mathrm{LE}}$ mixing, due to the large energetic gap between the ${ }^{3} \mathrm{~T}_{\mathrm{LE}}$ and ${ }^{3} \mathrm{~T}_{\mathrm{CR}}$ diabats in the strongly singlet-triplet exchange- 
split regime that encompasses SF systems, relative to the stronger ${ }^{1} \mathrm{TT}$ mixing with energetically proximal ${ }^{1} \mathrm{~S}_{\mathrm{CR}}$, as well as ${ }^{1} \mathrm{~S}_{\mathrm{LE}}$ diabats, according to the following interaction matrix:

$$
\begin{array}{ccccc}
S_{L E} & \left(l_{A} h_{A} \mid h_{B} l_{B}\right) & \left(l_{A} \mid l_{B}\right) & \left(h_{B} \mid h_{A}\right) & \left(l_{A} l_{B} \mid h_{B} l_{A}\right) \\
\left(l_{B} h_{B} \mid h_{A} l_{A}\right) & S_{L E} & \left(h_{A} \mid h_{B}\right) & \left(l_{B} \mid l_{A}\right) & \left(l_{B} l_{A} \mid h_{A} l_{B}\right) \\
\left(l_{A} \mid l_{B}\right) & \left(h_{A} \mid h_{B}\right) & S_{C R} & \left(h_{A} h_{B} \mid l_{A} l_{B}\right) & \left(h_{B} \mid l_{A}\right) \\
\left(h_{B} \mid h_{A}\right) & \left(l_{B} \mid l_{A}\right) & \left(h_{A} h_{B} \mid l_{A} l_{B}\right) & S_{C R} & \left(h_{A} \mid l_{B}\right) \\
& & & &
\end{array}
$$

The diabatic configurations along the diagonal of the above symmetric interaction matrix are as follows: $\mathrm{A}^{*} \mathrm{~B}^{0}, \mathrm{~A}^{0} \mathrm{~B}^{*},\left(\mathrm{~A}^{+} \mathrm{B}^{-}\right)^{\mathrm{s}},\left(\mathrm{A}^{-} \mathrm{B}^{+}\right)^{\mathrm{s}},\left(\mathrm{A}^{\mathrm{t}} \mathrm{B}^{\mathrm{t}}\right)^{\mathrm{s}}$. Here ${ }^{*}$ denotes a singlet local excitation, and ${ }^{\mathrm{s}}$ denotes net singlet spin. Again, let us assume that the diagonal diabatic energies have been corrected for orbital nonorthogonality between the two monomers of dimer AB. When applied to $\mathrm{S}_{\mathrm{TT}}$, this correction corresponds to the energetic gap from the noninteracting ${ }^{5} \mathrm{TT}$ state to the noninteracting and nonoverlapping T...T state. When configuration-interaction is turned on through diagonalization of the above interaction matrix containing the diagonally corrected $\mathrm{S}_{\mathrm{TT}}$ ( $\left.{ }^{1} \mathrm{TT}\right)$ diabat, ${ }^{1} \mathrm{TT}$ will be stabilized in SF systems by mixing with higher-lying $\mathrm{S}_{\mathrm{CR}}$ configurations (through leading terms involving one-electron coupling) in addition to quasidegenerate $\mathrm{S}_{\mathrm{LE}}$ configurations (through smaller two-electron-coupling terms). Indirect $\mathrm{S}_{\mathrm{LE}}{ }^{-}$ $\mathrm{S}_{\mathrm{TT}}(\mathrm{LE}-\mathrm{TT})$ mixing through one-electron coupling $(\mathrm{V})$ to $\mathrm{S}_{\mathrm{CR}}(\mathrm{CT})$ intermediates predominates in SF systems and takes the form from second-order perturbation theory3:

$$
\frac{\langle L E|V| C T\rangle\langle C T|V| T T\rangle}{E_{C T}-E_{T T}}
$$

Now having illustrated the source of the energetic stabilization of ${ }^{1} \mathrm{TT}$ relative to ${ }^{5} \mathrm{TT}$, let us now examine where this splitting leaves the pair-state energies relative to the sum of the lowest-lying triplet energies. The computed $\mathrm{T}_{1}$ and $\mathrm{T}_{2}$ adiabatic triplet states, which will adopt predominantly $\mathrm{T}_{\mathrm{LE}}$ character, will be marginally stabilized by electronic coupling to the higher- 
lying $\mathrm{T}_{\mathrm{CR}}$ configurations (and the adiabats with predominantly $\mathrm{T}_{\mathrm{CR}}$ character will, in turn, be concomitantly destabilized to conserve total energy). $T_{1}$ and $T_{2}$ are split from one another by Dexter exchange, but the summation $\left(\mathrm{T}_{1}+\mathrm{T}_{2}\right)$ will be somewhat less than $\left(\mathrm{T}_{\mathrm{LE}}+\mathrm{T}_{\mathrm{LE}}={ }^{5} \mathrm{TT}\right)$ due to the stabilization conferred by mixing with $\mathrm{T}_{\mathrm{CR}}$ when this configuration-interaction is relevant. Based on the results of the slip-scan over TIPS-tetracene dimer-1 geometries, the $T_{\mathrm{CR}}$ admixture is irrelevant, and a tight energetic convergence between $\left(T_{1}+T_{2}\right)$ and ${ }^{5} \mathrm{TT}$ (likely with subtle triplet-energy overestimations due to the lack of dynamic correlation in the electronic-structure methodology) is observed. In the covalently coupled systems, however, the expected ${ }^{1} \mathrm{TT}<\mathrm{T}_{1}+$ $\mathrm{T}_{2}<{ }^{5} \mathrm{TT}$ relationship does indeed manifest. These results are summarized in Figure S3.

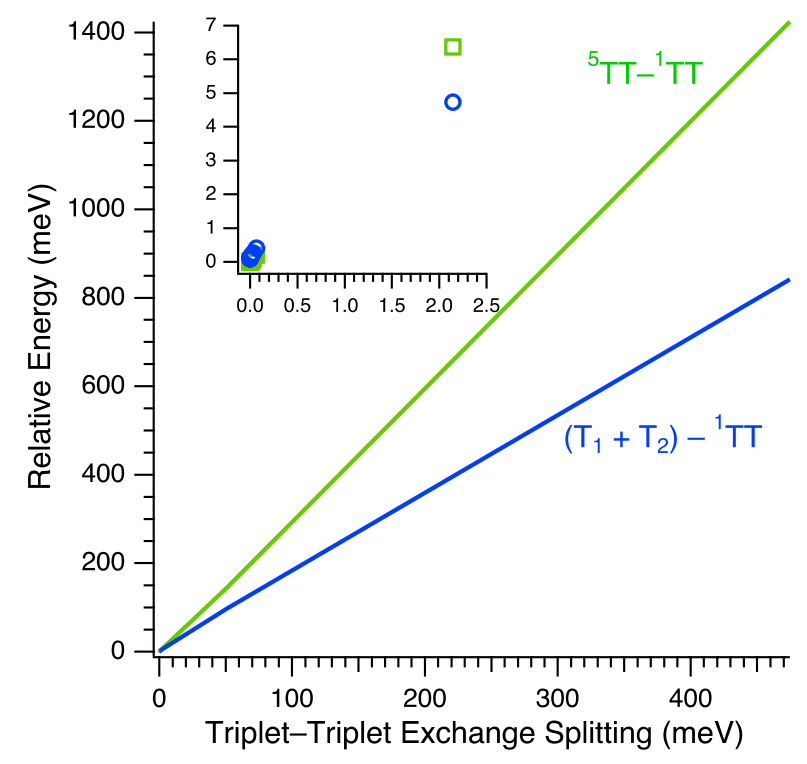

Figure S3. Comparison of interaction energy — the stabilization of ${ }^{1} \mathrm{TT}$ relative to ${ }^{5} \mathrm{TT}-$ and the stabilization of ${ }^{1} \mathrm{TT}$ relative to $\left(\mathrm{T}_{1}+\mathrm{T}_{2}\right)$, which itself is a metric for interaction-driven stabilization of the coupled monomeric triplet states. The energetic relationship ${ }^{1} \mathrm{TT}<\mathrm{T}_{1}+\mathrm{T}_{2}<$ ${ }^{5} \mathrm{TT}$ is observed generally across the studied SF systems, with the data underlying this figure comprising the slip-scan of TIPS-tetracene dimer 1, bipentacene, $\mathrm{BTDO}_{1}$ and neurosporene. Note that the methodology applied to neurosporene was not DMRG $(4,4)-\mathrm{SCF}$ but rather 
DMRG(18,18)-SCF/NEVPT2, and the triplet-triplet exchange splitting was gleaned as one-third the energetic difference between ${ }^{5} \mathrm{TT}$ and ${ }^{1} \mathrm{TT}$, since the ${ }^{3} \mathrm{TT}$ state was not calculated among the states that were averaged over the orbital optimization. The inset displays the results for the weak-coupling regime encompassing TIPS-tetracene dimer 1 (all geometries along the scan) and bipentacene. Only for bipentacene is there a distinguishable energetic gap from $\left(\mathrm{T}_{1}+\mathrm{T}_{2}\right)$ to ${ }^{5} \mathrm{TT}$ in this regime.

Because the internal coordinates of TIPS-tetracene dimer 1 are constant throughout the scan over separation length, the relative decrease in the exciton-splitting quantities reflects the approach toward the static-disorder baseline of triplet-triplet splitting caused by the utilization of crystal-structure geometries for the excited-state calculations. On the other hand, the internal coordinates of the $\mathrm{BTDO}_{1}$, bipentacene and neurosporene intramolecular-singlet-fission systems are geometrically optimized and, in turn, should feature triplet energies that are uncontaminated by this static disorder. Therefore, Davydov splitting may be compared between these three different systems. As presented in Table S4, the trend observed for exchange splitting is replicated in the trends observed for Davydov splitting between the triplets and between the triplet pairs.

Table S4. As in Table S3 For the Intramolecular-Singlet-Fission Systems.

\begin{tabular}{|l|l|l|}
\hline Intramolecular-SF System & $\left(\mathrm{T}_{2}-\mathrm{T}_{1}\right)$ Relative Energy & Ditto $\left(\mathrm{T}_{1}+\mathrm{T}_{2}\right)-{ }^{1} \mathrm{TT}$ \\
\hline BTDO $_{1}$ & $64.0 \mathrm{meV}$ & $96.4 \mathrm{meV}$ \\
\hline Bipentacene & $7.11 \mathrm{meV}$ & $4.73 \mathrm{meV}$ \\
\hline Neurosporene & $841 \mathrm{meV}$ & $783 \mathrm{meV}$ \\
\hline
\end{tabular}


Figure S4 summarizes the trends observed between the energetic quantities of interest for singlet-fission systems in the context of characterizing TT: $J,\left(\mathrm{~T}_{2}-\mathrm{T}_{1}\right)$ and $\left(\mathrm{T}_{1}+\mathrm{T}_{2}\right)-{ }^{1} \mathrm{TT}$.

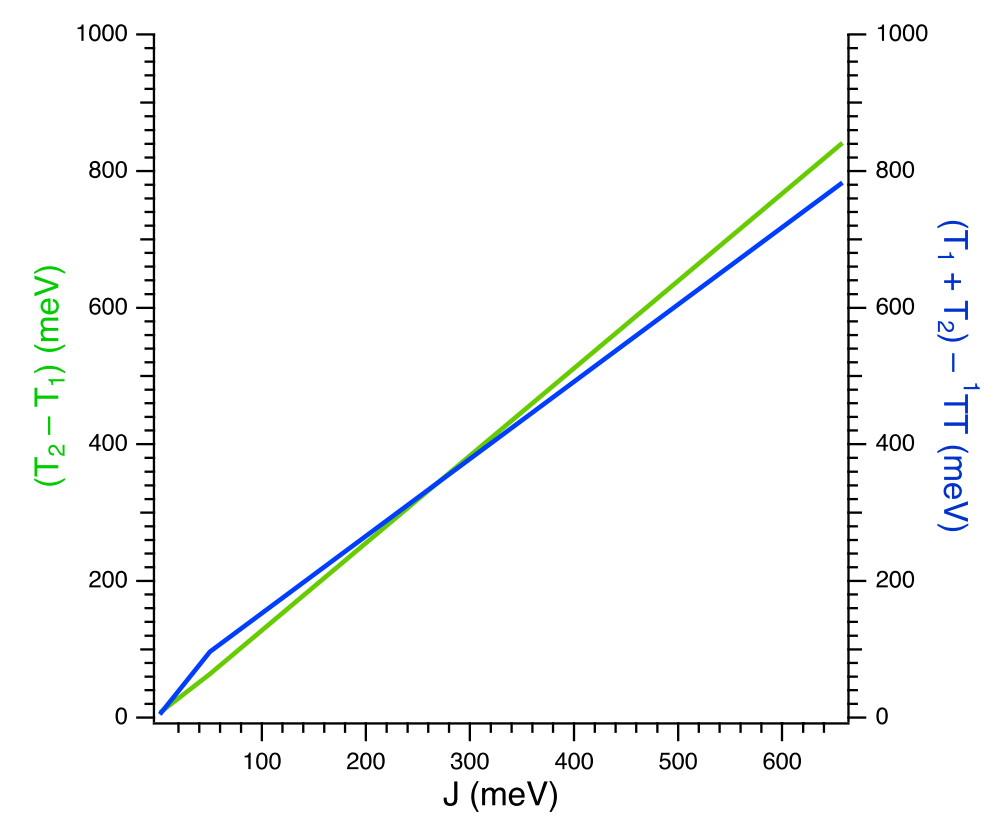

Figure S4. Summary of the direct proportionality between the Davydov-splitting quantities (vertical axes) and $J$.

We end with a discussion of the manifold of TT states inherent to carotenoids. Moving to such a strongly overlapping system leads to a large increase in $J$ at the DMRG $(4,4)-\mathrm{SCF}$ level of theory, along with a large residual error. That is, the magnitude of $J$ and its residual error grow monotonically when progressing from weakly to strongly overlapping systems. Specifically, the energetic gap from ${ }^{1} \mathrm{TT}$ to ${ }^{3} \mathrm{TT}$ increases faster than that between ${ }^{1} \mathrm{TT}$ and ${ }^{5} \mathrm{TT}$, causing $3\left({ }^{3} \mathrm{TT}\right)-{ }^{5} \mathrm{TT}$ to become increasingly more positive. This increase should be attributed to the lack of dynamic correlation, as well as the lack of static correlation beyond the minimal active space, at the DMRG(4,4)-SCF level of theory. As ${ }^{1} \mathrm{TT}$ states become less diabatically TT in character - that is, more configurationally mixed - their proper energetic resolution with respect to the ${ }^{3} \mathrm{TT}$ and ${ }^{5} \mathrm{TT}$ states relies on the incorporation of outlying-correlation effects. As illustrated in Figure S5, we may more satisfactorily resolve the exchange-split ladder of the low- 
lying TT states of the carotenoid neurosporene by turning to a DMRG $(6,6)-\mathrm{SCF}$ spin-stateaveraged calculation, with the converged $(4,4)$ orbitals as the initial guess, in which four singlets (incorporating the ${ }^{1} 2 \mathrm{~B}_{\mathrm{u}}{ }^{1} \mathrm{TT}$ state), three triplets and one quintet are converged.

(a)

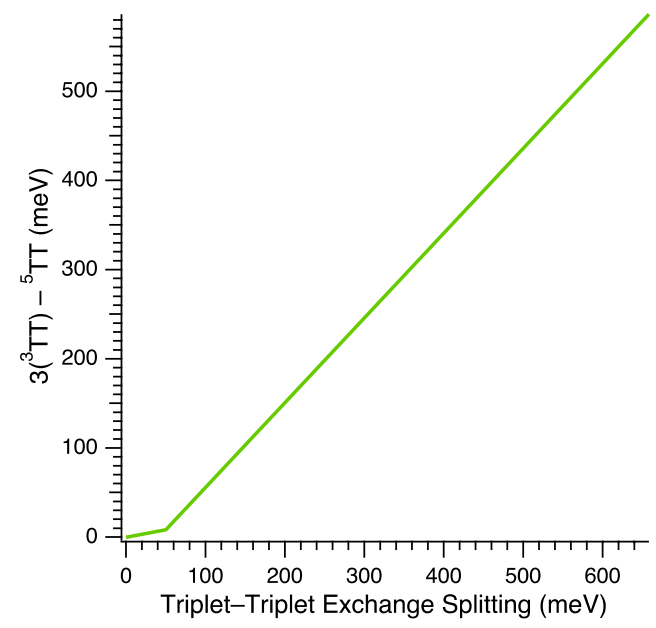

(b)

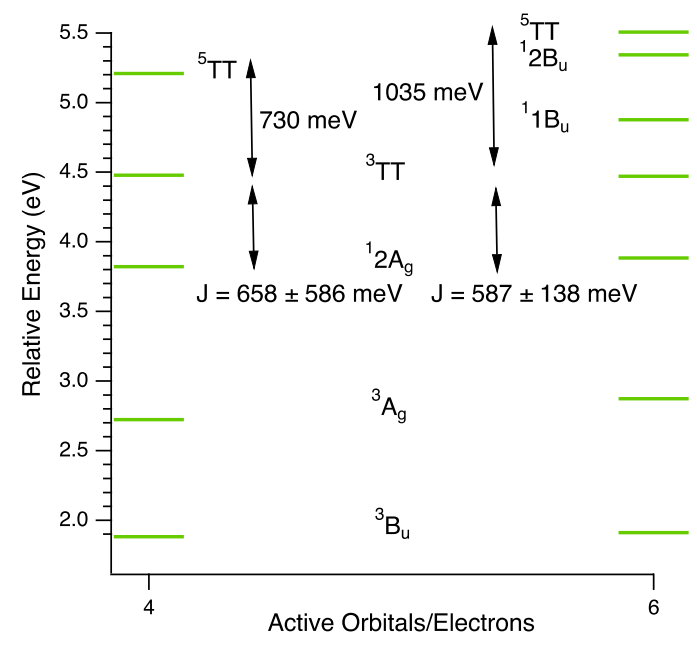

Figure S5. (a) For TIPS-tetracene dimer 1, bipentacene, $\mathrm{BTDO}_{1}$ and neurosporene at the spinstate-averaged (two singlets, three triplets, one quintet) DMRG(4,4)-SCF level of theory, the correlation between the following energetic relations: ${ }^{3} \mathrm{TT}-{ }^{1} \mathrm{TT}$ (the triplet-triplet exchange splitting, $J$ ) and $3\left({ }^{3} \mathrm{TT}\right)-{ }^{5} \mathrm{TT}$ (the residual error, or $\left.3(J)-3 J\right)$. (b) Comparison between the state-manifolds computed at the spin-state-averaged DMRG-SCF level of theory with 4 and 6 active orbitals/electrons, respectively. For the DMRG(6,6)-SCF calculation, the number of singlet states included in the state-averaging was increased from 2 (resolving the predominant configurations of $1 \mathrm{~A}_{\mathrm{g}}$ and $2 \mathrm{~A}_{\mathrm{g}}$ ) to 4 (resolving the predominant configurations of $1 \mathrm{~A}_{\mathrm{g}}, 2 \mathrm{~A}_{\mathrm{g}}, 1 \mathrm{~B}_{\mathrm{u}}$ and $\left.2 \mathrm{~B}_{\mathrm{u}}\right) . J$ is energetically defined as ${ }^{3} \mathrm{TT}-{ }^{1} 2 \mathrm{~A}_{\mathrm{g}}$, and the error is $3(J)-\left({ }^{5} \mathrm{TT}-{ }^{1} 2 \mathrm{~A}_{\mathrm{g}}\right)$.

The results suggest that there is a limitation to the numerical DMRG(4,4)-SCF approach when resolving the exchange-split ladder of strongly interacting ${ }^{1} \mathrm{TT}$ states. As the results improve, however, with active-space expansion, the increased error in the numerically resolved $J$ should not be misconstrued as a breakdown in the theoretical triplet-triplet exchange splitting. 


\section{Table of Geometries}

TIPS-Tetracene Unit Cel1//Crystal-90K ${ }^{4}$

376

x14094

$\begin{array}{llll}\text { Si } & 12.493505 & 11.326382 & 10.038989\end{array}$

$\begin{array}{llll}\text { Si } & 3.558810 & 4.316441 & 6.928279\end{array}$

$\begin{array}{llll}\text { C } & 9.336035 & 8.384799 & 8.812674\end{array}$

$\begin{array}{llll}\text { C } & 8.438614 & 7.896621 & 9.805996\end{array}$

$\begin{array}{llll}\text { C } & 8.578531 & 8.237999 & 11.157692\end{array}$

$\begin{array}{llll}\mathrm{H} & 9.260270 & 8.850220 & 11.409961\end{array}$

$\begin{array}{llll}\text { C } & 7.749228 & 7.707016 & 12.140286\end{array}$

$\begin{array}{llll}\text { C } & 7.928938 & 8.005440 & 13.528020\end{array}$

H $\quad 8.593259 \quad 8.631677 \quad 13.790180$

$\begin{array}{llll}\text { C } & 7.164920 & 7.406030 & 14.473068\end{array}$

H $\quad 7.314466 \quad 7.597745 \quad 15.390960$

$\begin{array}{llll}\text { C } & 6.138629 & 6.491015 & 14.098938\end{array}$

$\mathrm{H} \quad 5.612242 \quad 6.073976 \quad 14.770763$

$\begin{array}{llll}\text { C } & 5.904254 & 6.207814 & 12.785962\end{array}$

H $\quad 5.201556 \quad 5.612776 \quad 12.551460$

$\begin{array}{llll}\text { C } & 6.704385 & 6.797276 & 11.756266\end{array}$

$\begin{array}{llll}\text { C } & 6.518868 & 6.499305 & 10.413790\end{array}$

H $\quad 5.800591 \quad 5.930793 \quad 10.162861$

$\begin{array}{llll}\text { C } & 7.363890 & 7.015065 & 9.415607\end{array}$ 

$\begin{array}{llll}\text { C } & 7.202023 & 6.678962 & 8.041953\end{array}$
$\begin{array}{llll}\text { C } & 8.122386 & 7.135188 & 7.076121\end{array}$
$\begin{array}{llll}\text { C } & 8.007252 & 6.774819 & 5.699282\end{array}$
H $\quad 7.287559 \quad 6.220173 \quad 5.422539$
$\begin{array}{llll}\text { C } & 8.907222 & 7.209342 & 4.776360\end{array}$
H $\quad 8.812764 \quad 6.957189 \quad 3.865340$
$\begin{array}{llll}\text { C } & 9.990017 & 8.042517 & 5.175131\end{array}$
H $\quad 10.619784 \quad 8.336268 \quad 4.527443$
$\begin{array}{llll}\text { C } & 10.131917 & 8.420218 & 6.469835\end{array}$
$\begin{array}{llll}\mathrm{H} & 10.859115 & 8.979838 & 6.713221\end{array}$
$\begin{array}{llll}\text { C } & 9.209713 & 7.993985 & 7.475226\end{array}$
$\begin{array}{llll}\text { C } & 10.381161 & 9.282783 & 9.197867\end{array}$
$\begin{array}{llll}\text { C } & 11.253374 & 10.056122 & 9.525399\end{array}$
$\begin{array}{llll}\text { C } & 12.800812 & 12.448787 & 8.558058\end{array}$
$\mathrm{H} \quad 13.471930 \quad 13.133654 \quad 8.842008$
C $\quad 13.398431 \quad 11.701522 \quad 7.363255$
H $\quad 14.221079 \quad 11.246652 \quad 7.640165$
$\mathrm{H} \quad 13.603633 \quad 12.339365 \quad 6.647849$
H $\quad 12.753937 \quad 11.040167 \quad 7.036730$
$\begin{array}{llll}\text { C } & 11.530375 & 13.200874 & 8.154259\end{array}$
$\mathrm{H} \quad 11.170670 \quad 13.676242 \quad 8.932523$
$\begin{array}{llll}\mathrm{H} & 10.863363 & 12.562429 & 7.824548\end{array}$
H $\quad 11.742799 \quad 13.845047 \quad 7.447401$ 


\begin{tabular}{|c|c|c|c|}
\hline $\mathrm{C}$ & 14.067284 & 10.465324 & 10.629015 \\
\hline $\mathrm{H}$ & 13.926517 & 10.233820 & 11.590992 \\
\hline C & 14.399940 & 9.157084 & 9.902378 \\
\hline 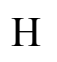 & 13.624875 & 8.559332 & 9.934897 \\
\hline & 15.164241 & 8.726630 & 10.338863 \\
\hline $\mathrm{H}$ & 14.623268 & 9.350607 & 8.967723 \\
\hline 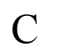 & 15.269179 & 11.422993 & 10.575377 \\
\hline $\mathrm{H}$ & 15.046700 & 12.253455 & 11.046224 \\
\hline & 15.484293 & 11.624956 & 9.641560 \\
\hline $\mathrm{H}$ & 16.042260 & 11.000980 & 11.004319 \\
\hline$\Gamma$ & 11.665618 & 12.282242 & 11.439630 \\
\hline $\mathrm{H}$ & 10.791139 & 12.610659 & 11.081424 \\
\hline C & 12.442382 & 13.518741 & 11.896062 \\
\hline $\mathrm{H}$ & 12.642060 & 14.083183 & 11.119977 \\
\hline $\mathrm{I}$ & 13.279332 & 13.239157 & 12.321820 \\
\hline & 11.901409 & 14.024403 & 12.538051 \\
\hline $\mathrm{C}$ & 11.338343 & 11.388629 & 12.634265 \\
\hline & 10.818046 & 10.615139 & 12.330201 \\
\hline $\mathrm{H}$ & 10.815214 & 11.897758 & 13.288993 \\
\hline $\mathrm{H}$ & 12.170479 & 11.080861 & 13.049295 \\
\hline $\mathrm{C}$ & 6.081558 & 5.877589 & 7.643015 \\
\hline & 5.116161 & 5.226784 & 7.330234 \\
\hline & 3.412946 & 4.223900 & 5.050421 \\
\hline
\end{tabular}




\begin{tabular}{llll}
$\mathrm{H}$ & 4.214492 & 3.727281 & 4.718531 \\
$\mathrm{C}$ & 3.444101 & 5.612474 & 4.402063 \\
$\mathrm{H}$ & 4.259809 & 6.081512 & 4.674950 \\
$\mathrm{H}$ & 2.659548 & 6.125220 & 4.690036 \\
$\mathrm{H}$ & 3.431356 & 5.517823 & 3.426173 \\
$\mathrm{C}$ & 2.178337 & 3.432776 & 4.602705 \\
$\mathrm{H}$ & 2.192216 & 2.544137 & 5.015220 \\
$\mathrm{H}$ & 2.186551 & 3.339933 & 3.627318 \\
$\mathrm{H}$ & 1.368011 & 3.908144 & 4.879447 \\
$\mathrm{C}$ & 3.699293 & 2.583776 & 7.659609 \\
$\mathrm{H}$ & 2.813910 & 2.135688 & 7.534564 \\
$\mathrm{C}$ & 4.744702 & 1.738694 & 6.928111 \\
$\mathrm{H}$ & 4.541625 & 1.725733 & 5.968984 \\
$\mathrm{H}$ & 4.727142 & 0.822926 & 7.278104 \\
$\mathrm{H}$ & 5.633484 & 2.125138 & 7.068578 \\
$\mathrm{C}$ & 3.981817 & 2.618441 & 9.160655 \\
$\mathrm{H}$ & 3.295404 & 3.154549 & 9.609712 \\
$\mathrm{H}$ & 4.863093 & 3.015887 & 9.318051 \\
$\mathrm{H}$ & 3.968080 & 1.706139 & 9.517520 \\
$\mathrm{C}$ & 2.129763 & 5.294156 & 7.668661 \\
$\mathrm{H}$ & 1.897654 & 6.003138 & 7.001529 \\
\hline & 0.879011 & 4.434304 & 7.836952 \\
$\mathrm{H}$ & 0.676924 & 3.981996 & 6.991472
\end{tabular}




$\begin{array}{llll}\mathrm{H} & 0.123206 & 5.003871 & 8.091066 \\ \mathrm{H} & 1.033797 & 3.764961 & 8.536938 \\ \mathrm{C} & 2.444434 & 6.017908 & 8.973758 \\ \mathrm{H} & 3.243006 & 6.572856 & 8.855417 \\ \mathrm{H} & 2.605734 & 5.359568 & 9.681789 \\ \mathrm{H} & 1.686647 & 6.586420 & 9.222507 \\ \mathrm{Si} & 8.748895 & 3.745518 & 1.657939 \\ \mathrm{Si} & 17.683590 & 10.755459 & -1.452771 \\ \mathrm{C} & 11.906365 & 6.687101 & 0.431624 \\ \mathrm{C} & 12.803786 & 7.175279 & 1.424946 \\ \mathrm{C} & 12.663869 & 6.833901 & 2.776642 \\ \mathrm{H} & 11.982130 & 6.221680 & 3.028911 \\ \mathrm{C} & 13.493172 & 7.364884 & 3.759236 \\ \mathrm{C} & 13.313462 & 7.066460 & 5.146970 \\ \mathrm{H} & 12.649141 & 6.440223 & 5.409130 \\ \mathrm{C} & 14.077480 & 7.665870 & 6.092018 \\ \mathrm{H} & 13.927934 & 7.474155 & 7.009910 \\ \mathrm{C} & 15.103771 & 8.580885 & 5.717888 \\ \mathrm{H} & 15.630158 & 8.997924 & 6.389713 \\ \mathrm{C} & 15.338146 & 8.864086 & 4.404912 \\ \mathrm{H} & 16.040844 & 9.459124 & 4.170410 \\ \mathrm{C} & 14.538015 & 8.274624 & 3.375216 \\ & 14.723532 & 8.572595 & 2.032740\end{array}$



H $\quad 15.441809 \quad 9.141107 \quad 1.781811$
$\begin{array}{llll}\text { C } & 13.878510 & 8.056835 & 1.034557\end{array}$
$\begin{array}{llll}\text { C } & 14.040377 & 8.392938 & -0.339097\end{array}$
$\begin{array}{llll}\text { C } & 13.120014 & 7.936712 & -1.304929\end{array}$
$\begin{array}{llll}\text { C } & 13.235148 & 8.297081 & -2.681768\end{array}$
$\mathrm{H} \quad 13.954841 \quad 8.851727 \quad-2.958511$
$\begin{array}{llll}\text { C } & 12.335178 & 7.862558 & -3.604690\end{array}$
H $\quad 12.429636 \quad 8.114711 \quad-4.515710$
$\begin{array}{llll}\text { C } & 11.252383 & 7.029383 & -3.205919\end{array}$
$\mathrm{H} \quad 10.622616 \quad 6.735632 \quad-3.853607$
$\begin{array}{llll}\text { C } & 11.110483 & 6.651682 & -1.911215\end{array}$
H $\quad 10.383285 \quad 6.092062 \quad-1.667829$
$\begin{array}{llll}\text { C } & 12.032687 & 7.077915 & -0.905824\end{array}$
$\begin{array}{llll}\text { C } & 10.861239 & 5.789117 & 0.816817\end{array}$
$\begin{array}{llll}\text { C } & 9.989026 & 5.015778 & 1.144349\end{array}$
$\begin{array}{llll}\text { C } & 8.441588 & 2.623113 & 0.177008\end{array}$
$\begin{array}{llll}\mathrm{H} & 7.770470 & 1.938246 & 0.460958\end{array}$
$\begin{array}{llll}\text { C } & 7.843969 & 3.370378 & -1.017795\end{array}$
H $\quad 7.021321 \quad 3.825248 \quad-0.740885$
$\mathrm{H} \quad 7.638767 \quad 2.732535 \quad-1.733201$
H $\quad 8.488463 \quad 4.031733 \quad-1.344320$
$\begin{array}{llll}\text { C } & 9.712025 & 1.871026 & -0.226791\end{array}$
$\mathrm{H} \quad 10.071730 \quad 1.395658 \quad 0.551473$ 

$\mathrm{H} \quad 10.379037 \quad 2.509471 \quad-0.556502$
H $\quad 9.499601 \quad 1.226853 \quad-0.933649$
$\begin{array}{llll}\text { C } & 7.175116 & 4.606576 & 2.247965\end{array}$
H $\quad 7.315883 \quad 4.838080 \quad 3.209942$
$\begin{array}{llll}\text { C } & 6.842460 & 5.914816 & 1.521328\end{array}$
$\mathrm{H} \quad 7.617525 \quad 6.512568 \quad 1.553847$
$\begin{array}{llll}\mathrm{H} & 6.078159 & 6.345270 & 1.957813\end{array}$
H $\quad 6.619132 \quad 5.721293 \quad 0.586674$
$\begin{array}{llll}\text { C } & 5.973221 & 3.648907 & 2.194327\end{array}$
$\begin{array}{llll}\mathrm{H} & 6.195700 & 2.818445 & 2.665174\end{array}$
H $\quad 5.758107 \quad 3.446944 \quad 1.260510$
$\begin{array}{llll}\mathrm{H} & 5.200140 & 4.070920 & 2.623269\end{array}$
$\begin{array}{llll}\text { C } & 9.576782 & 2.789658 & 3.058580\end{array}$
$\mathrm{H} \quad 10.451261 \quad 2.461241 \quad 2.700374$
$\begin{array}{llll}\text { C } & 8.800018 & 1.553159 & 3.515012\end{array}$
$\begin{array}{llll}\mathrm{H} & 8.600340 & 0.988717 & 2.738927\end{array}$
$\begin{array}{llll}\mathrm{H} & 7.963068 & 1.832743 & 3.940770\end{array}$
H $\quad 9.340991 \quad 1.047497 \quad 4.157001$
$\begin{array}{llll}\text { C } & 9.904057 & 3.683271 & 4.253215\end{array}$
$\mathrm{H} \quad 10.424354 \quad 4.456761 \quad 3.949151$
H $\quad 10.427186 \quad 3.174142 \quad 4.907943$
H $\quad 9.071921 \quad 3.991039 \quad 4.668245$
$\begin{array}{llll}\text { C } & 15.160842 & 9.194311 & -0.738035\end{array}$ 

$\begin{array}{llll}\text { C } & 16.126239 & 9.845116 & -1.050816\end{array}$
$\begin{array}{llll}\text { C } & 17.829454 & 10.848000 & -3.330629\end{array}$
H $\quad 17.027908 \quad 11.344619 \quad-3.662519$
$\begin{array}{llll}\text { C } & 17.798299 & 9.459426 & -3.978987\end{array}$
H $\quad 16.982591 \quad 8.990388 \quad-3.706100$
H $\quad 18.582852 \quad 8.946680 \quad-3.691014$
H $\quad 17.811044 \quad 9.554077 \quad-4.954877$
$\begin{array}{llll}\text { C } & 19.064063 & 11.639124 & -3.778345\end{array}$
H $\quad 19.050184 \quad 12.527763 \quad-3.365830$
H $\quad 19.055849 \quad 11.731967 \quad-4.753732$
H $\quad 19.874389 \quad 11.163756 \quad-3.501603$
$\begin{array}{llll}\text { C } & 17.543107 & 12.488124 & -0.721441\end{array}$
H $\quad 18.428490 \quad 12.936212 \quad-0.846486$
$\begin{array}{llll}\text { C } & 16.497698 & 13.333206 & -1.452939\end{array}$
H $\quad 16.700775 \quad 13.346167 \quad-2.412066$
H $\quad 16.515258 \quad 14.248974 \quad-1.102946$
$\mathrm{H} \quad 15.608916 \quad 12.946762 \quad-1.312472$
$\begin{array}{llll}\text { C } & 17.260583 & 12.453459 & 0.779605\end{array}$
H $\quad 17.946996 \quad 11.917351 \quad 1.228662$
H $\quad 16.379307 \quad 12.056013 \quad 0.937001$
$\mathrm{H} \quad 17.274320 \quad 13.365761 \quad 1.136470$
$\begin{array}{llll}\text { C } & 19.112637 & 9.777744 & -0.712389\end{array}$
$\begin{array}{llll}\mathrm{H} & 19.344746 & 9.068762 & -1.379521\end{array}$ 


\begin{tabular}{llll}
$\mathrm{C}$ & 20.363389 & 10.637596 & -0.544098 \\
$\mathrm{H}$ & 20.565476 & 11.089904 & -1.389578 \\
$\mathrm{H}$ & 21.119194 & 10.068029 & -0.289984 \\
$\mathrm{H}$ & 20.208603 & 11.306939 & 0.155888 \\
$\mathrm{C}$ & 18.797966 & 9.053992 & 0.592708 \\
$\mathrm{H}$ & 17.999394 & 8.499044 & 0.474367 \\
$\mathrm{H}$ & 18.636666 & 9.712332 & 1.300739 \\
$\mathrm{H}$ & 19.555753 & 8.485480 & 0.841457 \\
$\mathrm{Si}$ & 5.412705 & 11.281468 & 6.723111 \\
$\mathrm{Si}$ & -3.521990 & 18.291409 & 9.833821 \\
$\mathrm{C}$ & 2.255235 & 14.223051 & 7.949426 \\
$\mathrm{C}$ & 1.357814 & 14.711229 & 6.956104 \\
$\mathrm{C}$ & 1.497731 & 14.369851 & 5.604408 \\
$\mathrm{H}$ & 2.179470 & 13.757630 & 5.352139 \\
$\mathrm{C}$ & 0.668428 & 14.900834 & 4.621814 \\
$\mathrm{C}$ & 0.848138 & 14.602410 & 3.234080 \\
$\mathrm{H}$ & 1.512459 & 13.976173 & 2.971920 \\
$\mathrm{C}$ & 0.084120 & 15.201820 & 2.289032 \\
$\mathrm{H}$ & 0.233666 & 15.010105 & 1.371140 \\
$\mathrm{C}$ & -0.942171 & 16.116835 & 2.663162 \\
$\mathrm{H}$ & -1.468558 & 16.533874 & 1.991337 \\
\hline & -1.176546 & 16.400036 & 3.976138 \\
$\mathrm{H}$ & -1.879244 & 16.995074 & 4.210640
\end{tabular}



$\begin{array}{llll}\text { C } & -0.376415 & 15.810574 & 5.005834\end{array}$
$\begin{array}{llll}\text { C } & -0.561932 & 16.108545 & 6.348310\end{array}$
H $\quad-1.280209 \quad 16.677057 \quad 6.599239$
$\begin{array}{llll}\text { C } & 0.283090 & 15.592785 & 7.346493\end{array}$
$\begin{array}{llll}\text { C } & 0.121223 & 15.928888 & 8.720147\end{array}$
C $\quad \begin{array}{llll}1.041586 & 15.472662 & 9.685979\end{array}$
C $\quad 0.926452 \quad 15.833031 \quad 11.062818$
H $\quad 0.206759 \quad 16.387677 \quad 11.339561$
$\begin{array}{llll}\text { C } & 1.826422 & 15.398508 & 11.985740\end{array}$
$\mathrm{H} \quad 1.731964 \quad 15.650661 \quad 12.896760$
$\begin{array}{llll}\text { C } & 2.909217 & 14.565333 & 11.586969\end{array}$
$\begin{array}{llll}\mathrm{H} & 3.538984 & 14.271582 & 12.234657\end{array}$
$\begin{array}{llll}\text { C } & 3.051117 & 14.187632 & 10.292265\end{array}$
H $\quad 3.778315 \quad 13.628012 \quad 10.048879$
$\begin{array}{llll}\text { C } & 2.128913 & 14.613865 & 9.286874\end{array}$
$\begin{array}{llll}\text { C } & 3.300361 & 13.325067 & 7.564233\end{array}$
$\begin{array}{llll}\text { C } & 4.172574 & 12.551728 & 7.236701\end{array}$
$\begin{array}{llll}\text { C } & 5.720012 & 10.159063 & 8.204042\end{array}$
H $\quad 6.391130 \quad 9.474196 \quad 7.920092$
$\begin{array}{llll}\text { C } & 6.317631 & 10.906328 & 9.398845\end{array}$
H $\quad 7.140279 \quad 11.361198 \quad 9.121935$
$\mathrm{H} \quad \begin{array}{llll}6.522833 & 10.268485 & 10.114251\end{array}$
H $\quad 5.673137 \quad 11.567683 \quad 9.725370$ 


$\begin{array}{llll}\mathrm{C} & 4.449575 & 9.406976 & 8.607841 \\ \mathrm{H} & 4.089870 & 8.931608 & 7.829577 \\ \mathrm{H} & 3.782563 & 10.045421 & 8.937552 \\ \mathrm{H} & 4.661999 & 8.762803 & 9.314699 \\ \mathrm{C} & 6.986484 & 12.142526 & 6.133085 \\ \mathrm{H} & 6.845717 & 12.374030 & 5.171108 \\ \mathrm{C} & 7.319140 & 13.450766 & 6.859722 \\ \mathrm{H} & 6.544075 & 14.048518 & 6.827203 \\ \mathrm{H} & 8.083441 & 13.881220 & 6.423237 \\ \mathrm{H} & 7.542468 & 13.257243 & 7.794376 \\ \mathrm{C} & 8.188379 & 11.184857 & 6.186723 \\ \mathrm{H} & 7.965900 & 10.354395 & 5.715876 \\ \mathrm{H} & 8.403493 & 10.982894 & 7.120540 \\ \mathrm{H} & 8.961460 & 11.606870 & 5.757781 \\ \mathrm{C} & 4.584818 & 10.325608 & 5.322470 \\ \mathrm{H} & 3.710339 & 9.997191 & 5.680676 \\ \mathrm{C} & 5.361582 & 9.089109 & 4.866038 \\ \mathrm{H} & 5.561260 & 8.524667 & 5.642123 \\ \mathrm{H} & 6.198532 & 9.368693 & 4.440280 \\ \mathrm{H} & 4.820609 & 8.583447 & 4.224049 \\ \mathrm{C} & 4.257543 & 11.219221 & 4.127835 \\ \mathrm{H} & 3.737246 & 11.992711 & 4.431899 \\ & 3.734414 & 10.710092 & 3.473107\end{array}$




\begin{tabular}{llll}
$\mathrm{H}$ & 5.089679 & 11.526989 & 3.712805 \\
$\mathrm{C}$ & -0.999242 & 16.730261 & 9.119085 \\
$\mathrm{C}$ & -1.964639 & 17.381066 & 9.431866 \\
$\mathrm{C}$ & -3.667854 & 18.383950 & 11.711679 \\
$\mathrm{H}$ & -2.866308 & 18.880569 & 12.043569 \\
$\mathrm{C}$ & -3.636699 & 16.995376 & 12.360037 \\
$\mathrm{H}$ & -2.820991 & 16.526338 & 12.087150 \\
$\mathrm{H}$ & -4.421252 & 16.482630 & 12.072064 \\
$\mathrm{H}$ & -3.649444 & 17.090027 & 13.335927 \\
$\mathrm{C}$ & -4.902463 & 19.175074 & 12.159395 \\
$\mathrm{H}$ & -4.888584 & 20.063713 & 11.746880 \\
$\mathrm{H}$ & -4.894249 & 19.267917 & 13.134782 \\
$\mathrm{H}$ & -5.712789 & 18.699706 & 11.882653 \\
$\mathrm{C}$ & -3.381507 & 20.024074 & 9.102491 \\
$\mathrm{H}$ & -4.266890 & 20.472162 & 9.227536 \\
$\mathrm{C}$ & -2.336098 & 20.869156 & 9.833989 \\
$\mathrm{H}$ & -2.539175 & 20.882117 & 10.793116 \\
$\mathrm{H}$ & -2.353658 & 21.784924 & 9.483996 \\
$\mathrm{H}$ & -1.447316 & 20.482712 & 9.693522 \\
$\mathrm{H}$ & -3.098983 & 19.989409 & 7.601445 \\
$\mathrm{H}$ & -3.785396 & 19.453301 & 7.152388 \\
\hline & -2.217707 & 19.591963 & 7.444049 \\
\hline
\end{tabular}




\begin{tabular}{llll}
$\mathrm{C}$ & -4.951037 & 17.313694 & 9.093439 \\
$\mathrm{H}$ & -5.183146 & 16.604712 & 9.760571 \\
$\mathrm{C}$ & -6.201789 & 18.173546 & 8.925148 \\
$\mathrm{H}$ & -6.403876 & 18.625854 & 9.770628 \\
$\mathrm{H}$ & -6.957594 & 17.603979 & 8.671034 \\
$\mathrm{H}$ & -6.047003 & 18.842889 & 8.225162 \\
$\mathrm{C}$ & -4.636366 & 16.589942 & 7.788342 \\
$\mathrm{H}$ & -3.837794 & 16.034994 & 7.906683 \\
$\mathrm{H}$ & -4.475066 & 17.248282 & 7.080311 \\
$\mathrm{H}$ & -5.394153 & 16.021430 & 7.539593 \\
$\mathrm{Si}$ & 1.668095 & 3.790432 & 15.104161 \\
$\mathrm{Si}$ & 10.602790 & -3.219509 & 18.214871 \\
$\mathrm{C}$ & 4.825565 & 0.848849 & 16.330476 \\
$\mathrm{C}$ & 5.722986 & 0.360671 & 15.337154 \\
$\mathrm{C}$ & 5.583069 & 0.702049 & 13.985458 \\
$\mathrm{H}$ & 4.901330 & 1.314270 & 13.733189 \\
$\mathrm{C}$ & 6.412372 & 0.171066 & 13.002864 \\
$\mathrm{C}$ & 6.232662 & 0.469490 & 11.615130 \\
$\mathrm{H}$ & 5.568341 & 1.095727 & 11.352970 \\
$\mathrm{C}$ & 6.996680 & -0.129920 & 10.670082 \\
$\mathrm{H}$ & 6.847134 & 0.061795 & 9.752190 \\
$\mathrm{C}$ & 8.022971 & -1.044935 & 11.044212 \\
\hline
\end{tabular}



$\begin{array}{llll}\text { C } & 8.257346 & -1.328136 & 12.357188\end{array}$
H $\quad 8.960044 \quad-1.923174 \quad 12.591690$
$\begin{array}{llll}\text { C } & 7.457215 & -0.738674 & 13.386884\end{array}$
$\begin{array}{llll}\text { C } & 7.642732 & -1.036645 & 14.729360\end{array}$
$\begin{array}{llll}\mathrm{H} & 8.361009 & -1.605157 & 14.980289\end{array}$
$\begin{array}{llll}\text { C } & 6.797710 & -0.520885 & 15.727543\end{array}$
$\begin{array}{llll}\text { C } & 6.959577 & -0.856988 & 17.101197\end{array}$
$\begin{array}{llll}\text { C } & 6.039214 & -0.400762 & 18.067029\end{array}$
$\begin{array}{llll}\text { C } & 6.154348 & -0.761131 & 19.443868\end{array}$
$\begin{array}{llll}\mathrm{H} & 6.874041 & -1.315777 & 19.720611\end{array}$
$\begin{array}{llll}\text { C } & 5.254378 & -0.326608 & 20.366790\end{array}$
H $\quad 5.348836 \quad-0.578761 \quad 21.277810$
$\begin{array}{llll}\text { C } & 4.171583 & 0.506567 & 19.968019\end{array}$
H $\quad 3.541816 \quad 0.800318 \quad 20.615707$
$\begin{array}{llll}\text { C } & 4.029683 & 0.884268 & 18.673315\end{array}$
$\mathrm{H} \quad 3.302485 \quad 1.443888 \quad 18.429929$
$\begin{array}{llll}\text { C } & 4.951887 & 0.458035 & 17.667924\end{array}$
$\begin{array}{llll}\text { C } & 3.780439 & 1.746833 & 15.945283\end{array}$
$\begin{array}{llll}\text { C } & 2.908226 & 2.520172 & 15.617751\end{array}$
$\begin{array}{llll}\text { C } & 1.360788 & 4.912837 & 16.585092\end{array}$
H $\quad 0.689670 \quad 5.597704 \quad 16.301142$
$\begin{array}{llll}\text { C } & 0.763169 & 4.165572 & 17.779895\end{array}$
$\begin{array}{llll}\mathrm{H} & -0.059479 & 3.710702 & 17.502985\end{array}$ 

H $\quad 0.557967 \quad 4.803415 \quad 18.495301$
H $\quad 1.407663 \quad 3.504217 \quad 18.106420$
$\begin{array}{llll}\text { C } & 2.631225 & 5.664924 & 16.988891\end{array}$
$\begin{array}{llll}\mathrm{H} & 2.990930 & 6.140292 & 16.210627\end{array}$
$\begin{array}{llll}\mathrm{H} & 3.298237 & 5.026479 & 17.318602\end{array}$
$\begin{array}{llll}\mathrm{H} & 2.418801 & 6.309097 & 17.695749\end{array}$
$\begin{array}{llll}\text { C } & 0.094316 & 2.929374 & 14.514135\end{array}$
$\begin{array}{llll}\mathrm{H} & 0.235083 & 2.697870 & 13.552158\end{array}$
$\begin{array}{llll}\text { C } & -0.238340 & 1.621134 & 15.240772\end{array}$
$\begin{array}{llll}\mathrm{H} & 0.536725 & 1.023382 & 15.208253\end{array}$
$\begin{array}{llll}\mathrm{H} & -1.002641 & 1.190680 & 14.804287\end{array}$
$\begin{array}{llll}\mathrm{H} & -0.461668 & 1.814657 & 16.175427\end{array}$
$\begin{array}{llll}\text { C } & -1.107579 & 3.887043 & 14.567773\end{array}$
$\begin{array}{llll}\mathrm{H} & -0.885100 & 4.717505 & 14.096926\end{array}$
H $\quad-1.322693 \quad 4.089006 \quad 15.501590$
$\begin{array}{llll}\mathrm{H} & -1.880660 & 3.465030 & 14.138831\end{array}$
C $\quad 2.495982 \quad 4.746292 \quad 13.703520$
$\begin{array}{llll}\mathrm{H} & 3.370461 & 5.074709 & 14.061726\end{array}$
$\begin{array}{llll}\text { C } & 1.719218 & 5.982791 & 13.247088\end{array}$
$\mathrm{H} \quad 1.519540 \quad 6.547233 \quad 14.023173$
$\begin{array}{llll}\mathrm{H} & 0.882268 & 5.703207 & 12.821330\end{array}$
$\begin{array}{llll}\mathrm{H} & 2.260191 & 6.488453 & 12.605099\end{array}$
$\begin{array}{llll}\text { C } & 2.823257 & 3.852679 & 12.508885\end{array}$ 


$\begin{array}{llll}\mathrm{H} & 3.343554 & 3.079189 & 12.812949 \\ \mathrm{H} & 3.346386 & 4.361808 & 11.854157 \\ \mathrm{H} & 1.991121 & 3.544911 & 12.093855 \\ \mathrm{C} & 8.080042 & -1.658361 & 17.500135 \\ \mathrm{C} & 9.045439 & -2.309166 & 17.812916 \\ \mathrm{C} & 10.748654 & -3.312050 & 20.092729 \\ \mathrm{H} & 9.947108 & -3.808669 & 20.424619 \\ \mathrm{C} & 10.717499 & -1.923476 & 20.741087 \\ \mathrm{H} & 9.901791 & -1.454438 & 20.468200 \\ \mathrm{H} & 11.502052 & -1.410730 & 20.453114 \\ \mathrm{H} & 10.730244 & -2.018127 & 21.716977 \\ \mathrm{C} & 11.983263 & -4.103174 & 20.540445 \\ \mathrm{H} & 11.969384 & -4.991813 & 20.127930 \\ \mathrm{H} & 11.975049 & -4.196017 & 21.515832 \\ \mathrm{H} & 12.793589 & -3.627806 & 20.263703 \\ \mathrm{C} & 10.462307 & -4.952174 & 17.483541 \\ \mathrm{H} & 11.347690 & -5.400262 & 17.608586 \\ \mathrm{C} & 9.416898 & -5.797256 & 18.215039 \\ \mathrm{H} & 9.619975 & -5.810217 & 19.174166 \\ \mathrm{H} & 9.434458 & -6.713024 & 17.865046 \\ \mathrm{H} & 8.528116 & -5.410812 & 18.074572 \\ & 10.179783 & -4.917509 & 15.982495 \\ & 10.866196 & -4.381401 & 15.533438\end{array}$




\begin{tabular}{|c|c|c|c|c|}
\hline $\mathrm{H}$ & 9.298507 & -4.520063 & 15.825099 & \\
\hline $\mathrm{H}$ & 10.193520 & -5.829811 & $1 \quad 15.625630$ & \\
\hline $\mathrm{C}$ & 12.031837 & -2.241794 & $4 \quad 17.474489$ & \\
\hline $\mathrm{H}$ & 12.263946 & -1.532812 & $2 \quad 18.141621$ & \\
\hline $\mathrm{C}$ & 13.282589 & -3.101646 & $\begin{array}{ll}6 & 17.306198\end{array}$ & \\
\hline $\mathrm{H}$ & 13.484676 & -3.553954 & $\begin{array}{ll}4 & 18.151678\end{array}$ & \\
\hline $\mathrm{H}$ & 14.038394 & -2.532079 & $\begin{array}{ll}9 & 17.052084\end{array}$ & \\
\hline $\mathrm{H}$ & 13.127803 & -3.770989 & $9 \quad 16.606212$ & \\
\hline $\mathrm{C}$ & 11.717166 & -1.518042 & 216.169392 & \\
\hline $\mathrm{H}$ & 10.918594 & -0.963094 & $4 \quad 16.287733$ & \\
\hline $\mathrm{H}$ & 11.555866 & -2.176382 & 215.461361 & \\
\hline $\mathrm{H}$ & 12.474953 & -0.949530 & $\begin{array}{ll}0 & 15.920643\end{array}$ & \\
\hline \multicolumn{5}{|c|}{ Bipentacene//DFT(B3LYP)/cc-pVDZ } \\
\hline $\mathrm{Si}$ & \multicolumn{3}{|c|}{$5.23188500 \quad 6.15601500$} & 1.39645400 \\
\hline $\mathrm{C}$ & \multicolumn{2}{|c|}{6.43915900} & 1.94021900 & 0.33416700 \\
\hline $\mathrm{C}$ & \multicolumn{2}{|c|}{5.44316500} & 0.97946600 & -0.01156300 \\
\hline $\mathrm{C}$ & \multicolumn{2}{|c|}{4.07022300} & 1.31475400 & -0.03908100 \\
\hline $\mathrm{H}$ & \multicolumn{2}{|c|}{3.78023900} & 2.33716000 & 0.20767200 \\
\hline $\mathrm{C}$ & \multicolumn{2}{|c|}{3.08597000} & 0.38525600 & -0.37201000 \\
\hline $\mathrm{C}$ & \multicolumn{2}{|c|}{1.69524700} & 0.72751800 & -0.40192900 \\
\hline $\mathrm{H}$ & \multicolumn{2}{|c|}{1.42020500} & 1.74988300 & -0.13417600 \\
\hline $\mathrm{C}$ & \multicolumn{2}{|c|}{0.71983300} & -0.19563500 & -0.71618000 \\
\hline $\mathrm{C}$ & \multicolumn{2}{|c|}{1.13113000} & -1.54499500 & -1.03203300 \\
\hline
\end{tabular}




\begin{tabular}{|c|c|c|c|}
\hline $\mathrm{H}$ & 0.36989800 & -2.27419300 & -1.31402600 \\
\hline $\mathrm{C}$ & 2.44631000 & -1.91271500 & -1.02406700 \\
\hline $\mathrm{H}$ & 2.73443600 & -2.93462500 & -1.28245700 \\
\hline $\mathrm{C}$ & 3.48006000 & -0.97221000 & -0.69354400 \\
\hline $\mathrm{C}$ & 4.82793500 & -1.31459500 & -0.67632500 \\
\hline $\mathrm{H}$ & 5.12555600 & -2.33510300 & -0.92180300 \\
\hline $\mathrm{C}$ & 5.83686100 & -0.37884800 & -0.34606500 \\
\hline $\mathrm{C}$ & 6.05453200 & 3.26889000 & 0.66846700 \\
\hline $\mathrm{C}$ & 5.72353900 & 4.41557900 & 0.96140100 \\
\hline $\mathrm{C}$ & 6.43563400 & 6.72586400 & 2.78665700 \\
\hline $\mathrm{H}$ & 7.42548000 & 6.62917300 & 2.29922600 \\
\hline $\mathrm{C}$ & 6.45283300 & 5.81217500 & 4.02458900 \\
\hline $\mathrm{H}$ & 7.27860200 & 6.09039600 & 4.70334900 \\
\hline $\mathrm{H}$ & 6.58393500 & 4.75252100 & 3.75443400 \\
\hline $\mathrm{H}$ & 5.51854700 & 5.89546100 & 4.60401400 \\
\hline $\mathrm{C}$ & 6.26353900 & 8.20155700 & 3.19074300 \\
\hline $\mathrm{H}$ & 5.30481800 & 8.37170600 & 3.70903000 \\
\hline $\mathrm{H}$ & 6.29776300 & 8.88415700 & 2.32587100 \\
\hline $\mathrm{H}$ & 7.06354800 & 8.51277000 & 3.88577200 \\
\hline $\mathrm{C}$ & 5.49575000 & 7.23978900 & -0.17257900 \\
\hline $\mathrm{H}$ & 5.19595500 & 8.26467900 & 0.12133400 \\
\hline $\mathrm{C}$ & 4.59651000 & 6.80238600 & -1.34288800 \\
\hline $\mathrm{H}$ & 4.75777500 & 7.44765500 & -2.22441400 \\
\hline
\end{tabular}




\begin{tabular}{|c|c|c|c|}
\hline $\mathrm{H}$ & 3.52492700 & 6.85422100 & -1.09140600 \\
\hline $\mathrm{H}$ & 4.81658500 & 5.76578500 & -1.64999200 \\
\hline $\mathrm{C}$ & 6.97027800 & 7.28735700 & -0.61149500 \\
\hline $\mathrm{H}$ & 7.34178000 & 6.28187400 & -0.87318900 \\
\hline $\mathrm{H}$ & 7.62941000 & 7.69235000 & 0.17321900 \\
\hline $\mathrm{H}$ & 7.09371800 & 7.92528800 & -1.50439400 \\
\hline $\mathrm{C}$ & 2.72590300 & 7.48266400 & 2.05172700 \\
\hline $\mathrm{H}$ & 2.91250900 & 8.16905700 & 1.20971700 \\
\hline C & 3.35850800 & 6.09584300 & 1.83467700 \\
\hline $\mathrm{H}$ & 2.91196000 & 5.66817800 & 0.91568400 \\
\hline $\mathrm{C}$ & 3.01035100 & 5.14327000 & 2.99186100 \\
\hline $\mathrm{H}$ & 3.36594300 & 5.53444900 & 3.95941200 \\
\hline $\mathrm{H}$ & 3.11085500 & 7.96697400 & 2.96487400 \\
\hline $\mathrm{H}$ & 1.63096200 & 7.39884800 & 2.17003500 \\
\hline $\mathrm{H}$ & 1.91655800 & 5.01662900 & 3.07851000 \\
\hline $\mathrm{H}$ & 3.45328900 & 4.14409000 & 2.85441400 \\
\hline $\mathrm{Si}$ & 8.44374700 & -4.95280500 & -1.38816000 \\
\hline $\mathrm{C}$ & 7.21511100 & -0.74068600 & -0.33484200 \\
\hline $\mathrm{C}$ & 8.21247100 & 0.22125700 & 0.00757900 \\
\hline $\mathrm{C}$ & 9.58560500 & -0.11150800 & 0.02592300 \\
\hline $\mathrm{H}$ & 9.87668900 & -1.13105500 & -0.23119200 \\
\hline $\mathrm{C}$ & 10.56966400 & 0.81668500 & 0.36025200 \\
\hline C & 11.96579500 & 0.48053100 & 0.37960300 \\
\hline
\end{tabular}




\begin{tabular}{|c|c|c|c|}
\hline $\mathrm{H}$ & 12.25876600 & -0.54023900 & 0.12197800 \\
\hline $\mathrm{C}$ & 12.90896800 & 1.41519600 & 0.71267800 \\
\hline $\mathrm{H}$ & 13.96776700 & 1.14692100 & 0.72366100 \\
\hline $\mathrm{C}$ & 12.52096600 & 2.75344000 & 1.05025200 \\
\hline $\mathrm{H}$ & 13.29054700 & 3.48277800 & 1.31286300 \\
\hline $\mathrm{C}$ & 11.20134600 & 3.11751600 & 1.04471600 \\
\hline $\mathrm{H}$ & 10.90288100 & 4.13690800 & 1.30147400 \\
\hline $\mathrm{C}$ & 10.17696600 & 2.17098600 & 0.70174700 \\
\hline $\mathrm{C}$ & 8.82573400 & 2.51038200 & 0.68646200 \\
\hline $\mathrm{H}$ & 8.52644000 & 3.52774700 & 0.94273900 \\
\hline $\mathrm{C}$ & 7.81916000 & 1.57767100 & 0.34818900 \\
\hline $\mathrm{C}$ & 7.60048500 & -2.06973800 & -0.66819200 \\
\hline $\mathrm{C}$ & 7.93459300 & -3.21587000 & -0.95943900 \\
\hline $\mathrm{C}$ & 6.99202900 & -5.70618600 & -2.40343700 \\
\hline $\mathrm{H}$ & 6.13720500 & -5.60706300 & -1.70613200 \\
\hline $\mathrm{C}$ & 6.63162600 & -4.91690600 & -3.67415700 \\
\hline $\mathrm{H}$ & 5.68822800 & -5.28969000 & -4.11130700 \\
\hline $\mathrm{H}$ & 6.50542300 & -3.84141900 & -3.47223100 \\
\hline $\mathrm{H}$ & 7.40797400 & -5.02088300 & -4.45005300 \\
\hline $\mathrm{C}$ & 7.16205600 & -7.20469500 & -2.71334200 \\
\hline $\mathrm{H}$ & 7.98931300 & -7.38227300 & -3.42096700 \\
\hline $\mathrm{H}$ & 7.36528500 & -7.80300800 & -1.81018000 \\
\hline $\mathrm{H}$ & 6.24794800 & -7.61416100 & -3.17886000 \\
\hline
\end{tabular}




\begin{tabular}{|c|c|c|c|}
\hline $\mathrm{C}$ & 8.64929800 & -5.92025000 & 0.26324900 \\
\hline $\mathrm{H}$ & 8.95662900 & -6.94321000 & -0.02970200 \\
\hline $\mathrm{C}$ & 9.75849300 & -5.33144300 & 1.15339300 \\
\hline $\mathrm{H}$ & 9.86391000 & -5.91358100 & 2.08583600 \\
\hline $\mathrm{H}$ & 10.74142300 & -5.33133800 & 0.65530500 \\
\hline $\mathrm{H}$ & 9.53009000 & -4.29087700 & 1.44053600 \\
\hline $\mathrm{C}$ & 7.32825600 & -6.02983000 & 1.04571700 \\
\hline $\mathrm{H}$ & 6.94531300 & -5.03344500 & 1.32479900 \\
\hline $\mathrm{H}$ & 6.53954800 & -6.53966900 & 0.46934200 \\
\hline $\mathrm{H}$ & 7.47177400 & -6.60022900 & 1.98032500 \\
\hline $\mathrm{C}$ & 10.80438600 & -6.17353600 & -2.57387800 \\
\hline $\mathrm{H}$ & 10.87189900 & -6.81753700 & -1.68179800 \\
\hline $\mathrm{C}$ & 10.14767500 & -4.81374000 & -2.27333500 \\
\hline $\mathrm{H}$ & 10.77141000 & -4.30562700 & -1.51208100 \\
\hline $\mathrm{C}$ & 10.14111500 & -3.91514100 & -3.52232400 \\
\hline $\mathrm{H}$ & 9.58880800 & -4.38132700 & -4.35492800 \\
\hline $\mathrm{H}$ & 10.24708600 & -6.73266000 & -3.34427200 \\
\hline $\mathrm{H}$ & 11.83110900 & -6.03588000 & -2.95707900 \\
\hline $\mathrm{H}$ & 11.17079800 & -3.73583100 & -3.87955000 \\
\hline $\mathrm{H}$ & 9.67998300 & -2.93474100 & -3.32405000 \\
\hline $\mathrm{Si}$ & -8.42535000 & 4.90612100 & -1.58987300 \\
\hline $\mathrm{C}$ & -7.21348000 & 0.72964800 & -0.38589900 \\
\hline $\mathrm{C}$ & -8.21289100 & -0.21967800 & -0.01554500 \\
\hline
\end{tabular}




\begin{tabular}{|c|c|c|c|}
\hline $\mathrm{C}$ & -9.58561700 & 0.11524100 & -0.01107400 \\
\hline $\mathrm{H}$ & -9.87470700 & 1.12665200 & -0.30058500 \\
\hline $\mathrm{C}$ & -10.57175800 & -0.80093100 & 0.34925600 \\
\hline $\mathrm{C}$ & -11.96752600 & -0.46274400 & 0.35401500 \\
\hline $\mathrm{H}$ & -12.25853200 & 0.54965700 & 0.06326800 \\
\hline $\mathrm{C}$ & -12.91276500 & -1.38528400 & 0.71394200 \\
\hline $\mathrm{H}$ & -13.97125900 & -1.11558800 & $0.7134280 c$ \\
\hline $\mathrm{C}$ & -12.52733800 & -2.71255800 & 1.09497700 \\
\hline $\mathrm{H}$ & -13.29853000 & -3.43227900 & $1.3785580 \mathrm{C}$ \\
\hline $\mathrm{C}$ & -11.20816900 & -3.07818400 & 1.10453500 \\
\hline $\mathrm{H}$ & -10.91167700 & -4.08925700 & $1.3943370 \mathrm{C}$ \\
\hline $\mathrm{C}$ & -10.18168700 & -2.14426600 & 0.73433600 \\
\hline $\mathrm{C}$ & -8.83088800 & -2.48573500 & 0.73291100 \\
\hline $\mathrm{H}$ & -8.53354300 & -3.49478200 & 1.02225500 \\
\hline $\mathrm{C}$ & -7.82224900 & -1.56536100 & 0.36801400 \\
\hline $\mathrm{C}$ & -7.59584700 & 2.04846800 & -0.76077200 \\
\hline $\mathrm{C}$ & -7.92571000 & 3.18749700 & -1.08308700 \\
\hline $\mathrm{C}$ & -6.89248400 & 6.02791500 & -1.27720900 \\
\hline $\mathrm{H}$ & -6.11054700 & 5.53994500 & -1.89138300 \\
\hline $\mathrm{C}$ & -6.39160500 & 6.02337200 & 0.17767700 \\
\hline $\mathrm{H}$ & -5.41675100 & 6.53668200 & 0.25690600 \\
\hline $\mathrm{H}$ & -6.26499700 & 5.00165500 & 0.56940500 \\
\hline $\mathrm{H}$ & -7.08861800 & 6.55300600 & 0.84793600 \\
\hline
\end{tabular}




\begin{tabular}{|c|c|c|c|}
\hline C & -7.05739100 & 7.46665600 & -1.79996800 \\
\hline $\mathrm{H}$ & -7.81352600 & 8.02485400 & -1.22269900 \\
\hline $\mathrm{H}$ & -7.35943500 & 7.49975100 & -2.85949200 \\
\hline $\mathrm{H}$ & -6.10897400 & 8.02591500 & -1.71206500 \\
\hline C & -8.82050300 & 4.86028200 & -3.47329400 \\
\hline $\mathrm{H}$ & -9.11154500 & 5.89506600 & -3.73997400 \\
\hline $\mathrm{C}$ & -10.00774200 & 3.93700500 & -3.80079900 \\
\hline $\mathrm{H}$ & -10.21970100 & 3.93965600 & -4.88454000 \\
\hline $\mathrm{H}$ & -10.93266700 & 4.24332400 & -3.28611300 \\
\hline $\mathrm{H}$ & -9.79458300 & 2.89398300 & -3.51085700 \\
\hline C & -7.59138400 & 4.48271800 & -4.31959100 \\
\hline $\mathrm{H}$ & -7.22439300 & 3.47427900 & -4.06335900 \\
\hline $\mathrm{H}$ & -6.75436200 & 5.18607700 & -4.18210300 \\
\hline $\mathrm{H}$ & -7.84183800 & 4.47779200 & -5.39507200 \\
\hline $\mathrm{C}$ & -10.66846700 & 6.66167700 & -0.99596400 \\
\hline $\mathrm{H}$ & -10.83663100 & 6.74741400 & -2.08199600 \\
\hline $\mathrm{C}$ & -10.02504000 & 5.31965200 & -0.60174400 \\
\hline $\mathrm{H}$ & -10.71638000 & 4.51482200 & -0.91966800 \\
\hline $\mathrm{C}$ & -9.87414500 & 5.20613700 & 0.92532800 \\
\hline $\mathrm{H}$ & -9.24673800 & 6.01602800 & 1.33279300 \\
\hline $\mathrm{H}$ & -10.04383600 & 7.51749000 & -0.68912100 \\
\hline $\mathrm{H}$ & -11.64836300 & 6.78604000 & -0.50172900 \\
\hline $\mathrm{H}$ & -10.85819400 & 5.28083300 & 1.42141300 \\
\hline
\end{tabular}




\begin{tabular}{|c|c|c|c|}
\hline $\mathrm{H}$ & -9.41921400 & 4.24916500 & 1.22602900 \\
\hline $\mathrm{Si}$ & -5.23260500 & -6.10460500 & 1.57956200 \\
\hline $\mathrm{C}$ & -6.44270000 & -1.92976700 & 0.36925200 \\
\hline $\mathrm{C}$ & -5.44441700 & -0.98118200 & -0.00268700 \\
\hline $\mathrm{C}$ & -4.07149600 & -1.31755500 & -0.01208400 \\
\hline $\mathrm{H}$ & -3.78294600 & -2.33035600 & 0.27292900 \\
\hline $\mathrm{C}$ & -3.08529900 & -0.40047700 & -0.37257300 \\
\hline $\mathrm{C}$ & -1.69451700 & -0.74366900 & -0.38326100 \\
\hline $\mathrm{H}$ & -1.42098200 & -1.75567100 & -0.07730100 \\
\hline $\mathrm{C}$ & -0.71738400 & 0.16733400 & -0.72633000 \\
\hline $\mathrm{C}$ & -1.12688800 & 1.50416500 & -1.09359500 \\
\hline $\mathrm{H}$ & -0.36418900 & 2.22243900 & -1.39869400 \\
\hline $\mathrm{C}$ & -2.44197500 & 1.87210400 & -1.10504800 \\
\hline $\mathrm{H}$ & -2.72861200 & 2.88395900 & -1.40182800 \\
\hline $\mathrm{C}$ & -3.47743200 & 0.94459200 & -0.74455700 \\
\hline $\mathrm{C}$ & -4.82509000 & 1.28821500 & -0.74389300 \\
\hline $\mathrm{H}$ & -5.12128700 & 2.29893100 & -1.02843300 \\
\hline $\mathrm{C}$ & -5.83573800 & 0.36560800 & -0.38337100 \\
\hline $\mathrm{C}$ & -6.06038900 & -3.24798200 & 0.74501400 \\
\hline $\mathrm{C}$ & -5.73068200 & -4.38677200 & 1.06865000 \\
\hline $\mathrm{C}$ & -6.76392400 & -7.22716200 & 1.26221400 \\
\hline $\mathrm{H}$ & -7.54843900 & -6.73833000 & 1.87239900 \\
\hline $\mathrm{C}$ & -7.25881200 & -7.22482600 & -0.19472500 \\
\hline
\end{tabular}




\begin{tabular}{|c|c|c|c|}
\hline $\mathrm{H}$ & -8.23321900 & -7.73842500 & -0.27725200 \\
\hline $\mathrm{H}$ & -7.38408900 & -6.20368000 & -0.58834800 \\
\hline $\mathrm{H}$ & -6.55891300 & -7.75529900 & -0.86133100 \\
\hline $\mathrm{C}$ & -6.60105300 & -8.66508100 & 1.78785000 \\
\hline $\mathrm{H}$ & -5.84258500 & -9.22415300 & 1.21449400 \\
\hline $\mathrm{H}$ & -6.30324400 & -8.69651700 & 2.84862300 \\
\hline $\mathrm{H}$ & -7.54908700 & -9.22450000 & 1.69696400 \\
\hline C & -4.84416900 & -6.05652600 & 3.46424400 \\
\hline $\mathrm{H}$ & -4.55365600 & -7.09093400 & 3.73299700 \\
\hline C & -3.65853800 & -5.13235100 & 3.79506000 \\
\hline $\mathrm{H}$ & -3.45046500 & -5.13382300 & 4.87952600 \\
\hline $\mathrm{H}$ & -2.73152700 & -5.43865900 & 3.28417100 \\
\hline $\mathrm{H}$ & -3.87103300 & -4.08970700 & 3.50335200 \\
\hline $\mathrm{C}$ & -6.07656800 & -5.67872000 & 4.30566000 \\
\hline $\mathrm{H}$ & -6.44302000 & -4.67066900 & 4.04714500 \\
\hline $\mathrm{H}$ & -6.91279500 & -6.38257800 & 4.16581500 \\
\hline $\mathrm{H}$ & -5.82994100 & -5.67262400 & 5.38201200 \\
\hline $\mathrm{C}$ & -2.98757000 & -7.86095000 & 0.99530000 \\
\hline $\mathrm{H}$ & -2.82383000 & -7.94608300 & 2.08202900 \\
\hline $\mathrm{C}$ & -3.62905100 & -6.51896700 & 0.59783900 \\
\hline $\mathrm{H}$ & -2.93881400 & -5.71420900 & 0.91832600 \\
\hline $\mathrm{C}$ & -3.77382400 & -6.40622800 & -0.92986500 \\
\hline & -4.39981500 & -7.21611800 & -1.33948100 \\
\hline
\end{tabular}




$$
\begin{array}{llll}
\mathrm{H} & -3.61117700 & -8.71674700 & 0.68634000 \\
\mathrm{H} & -2.00564000 & -7.98584900 & 0.50521100 \\
\mathrm{H} & -2.78784300 & -6.48167300 & -1.42213000 \\
\mathrm{H} & -4.22747800 & -5.44937100 & -1.23294200
\end{array}
$$

$\mathrm{BTDO}_{1} / / \mathrm{DFT}(\mathrm{B} 3 \mathrm{LYP}) / \mathrm{cc}-\mathrm{pVDZ}$

C $\quad-13.63050600 \quad 1.03077500 \quad-0.97212500$

S $\quad-12.99740900 \quad-0.31478500 \quad 0.08644600$

$\mathrm{H} \quad-13.33241300 \quad 0.87967500 \quad-2.01956400$

$\mathrm{H} \quad-13.28740800 \quad 2.00996700 \quad-0.60844700$

H $\quad-14.72485900 \quad 0.97351500 \quad-0.89189200$

C $\quad-11.25726200 \quad-0.11675900 \quad-0.11345800$

$\begin{array}{llll}\text { C } & -10.53606300 & 0.78500100 & -0.87287100\end{array}$

$\begin{array}{llll}\text { C } & -9.13312800 & 0.61147300 & -0.76000900\end{array}$

$\begin{array}{llll}\text { C } & -8.76117700 & -0.42033300 & 0.08503700\end{array}$

S $\quad-10.19501700 \quad-1.20111800 \quad 0.76596300$

H $\quad-10.99251200 \quad 1.54948200 \quad-1.49842700$

$\mathrm{H} \quad-8.40441800 \quad 1.21517400 \quad-1.29997100$

$\begin{array}{llll}\text { C } & -7.44263200 & -0.86587600 & 0.41000500\end{array}$

S $\quad-6.00543100 \quad 0.13676300 \quad-0.13985800$

$\begin{array}{llll}\text { O } & -5.96521800 & 1.42365500 & 0.60046500\end{array}$

O $\quad-5.91460800 \quad 0.15831800 \quad-1.62548600$

C $\quad-4.80548100 \quad-1.04458400 \quad 0.58089100$

$\begin{array}{llll}\text { C } & -6.96070400 & -1.93110400 & 1.10543000\end{array}$ 


\begin{tabular}{|c|c|c|c|}
\hline $\mathrm{C}$ & -5.51347500 & -2.02647900 & 1.19969000 \\
\hline $\mathrm{H}$ & -7.60593200 & -2.68620300 & 1.55681200 \\
\hline $\mathrm{H}$ & -5.03604400 & -2.85289500 & 1.72854200 \\
\hline $\mathrm{C}$ & -3.40261700 & -0.78441900 & 0.45775400 \\
\hline S & -2.21507900 & -1.73271300 & 1.37940000 \\
\hline $\mathrm{C}$ & -2.80584600 & 0.17216100 & -0.33486300 \\
\hline $\mathrm{C}$ & -1.38041700 & 0.19972400 & -0.22642000 \\
\hline $\mathrm{H}$ & -3.37611300 & 0.81378900 & -1.00375400 \\
\hline $\mathrm{C}$ & -0.89573500 & -0.81667100 & 0.65383600 \\
\hline $\mathrm{C}$ & -0.45777900 & 1.04467700 & -0.88741400 \\
\hline $\mathrm{C}$ & 0.45779800 & -1.04473100 & 0.88720600 \\
\hline $\mathrm{C}$ & 0.89575500 & 0.81661100 & -0.65404900 \\
\hline $\mathrm{C}$ & 1.38043700 & -0.19978100 & 0.22620600 \\
\hline $\mathrm{O}$ & -0.83994600 & 2.00811300 & -1.78330600 \\
\hline $\mathrm{C}$ & -1.56739700 & 3.14802000 & -1.26533300 \\
\hline $\mathrm{C}$ & -0.65170900 & 4.19377300 & -0.63951500 \\
\hline $\mathrm{H}$ & -2.32922700 & 2.81428400 & -0.54121100 \\
\hline $\mathrm{H}$ & -2.09109300 & 3.55903200 & -2.14181100 \\
\hline $\mathrm{C}$ & -1.42740500 & 5.42146800 & -0.14771200 \\
\hline $\mathrm{H}$ & 0.10480900 & 4.49633100 & -1.38451800 \\
\hline $\mathrm{H}$ & -0.10148000 & 3.73915400 & 0.20304000 \\
\hline $\mathrm{C}$ & -0.53109600 & 6.49518800 & 0.47836100 \\
\hline $\mathrm{H}$ & -2.18749500 & 5.10535600 & 0.59096300 \\
\hline
\end{tabular}




\begin{tabular}{|c|c|c|c|}
\hline $\mathrm{H}$ & -1.99059500 & 5.86352300 & -0.99126800 \\
\hline $\mathrm{C}$ & -1.30019400 & 7.72499700 & 0.97232300 \\
\hline $\mathrm{H}$ & 0.22959600 & 6.81219700 & -0.26006700 \\
\hline $\mathrm{H}$ & 0.03237100 & 6.05355900 & 1.32183600 \\
\hline $\mathrm{C}$ & -0.39803200 & 8.79247900 & 1.59571000 \\
\hline $\mathrm{H}$ & -2.05976300 & 7.40638000 & 1.70988000 \\
\hline $\mathrm{H}$ & -1.86400300 & 8.16436700 & 0.12856700 \\
\hline $\mathrm{H}$ & 0.15254700 & 8.39341100 & 2.46472600 \\
\hline $\mathrm{H}$ & -0.97956900 & 9.66256400 & 1.94075100 \\
\hline $\mathrm{H}$ & 0.35021700 & 9.15734000 & 0.87127600 \\
\hline $\mathrm{O}$ & 0.83996600 & -2.00816000 & 1.78310400 \\
\hline $\mathrm{C}$ & 1.56739600 & -3.14808300 & 1.26513500 \\
\hline $\mathrm{C}$ & 0.65166600 & -4.19389500 & 0.63948000 \\
\hline $\mathrm{H}$ & 2.09119600 & -3.55902600 & 2.14158400 \\
\hline $\mathrm{H}$ & 2.32913900 & -2.81437400 & 0.54091000 \\
\hline $\mathrm{C}$ & 1.42734800 & -5.42160300 & 0.14768700 \\
\hline $\mathrm{H}$ & 0.10134600 & -3.73934400 & -0.20305200 \\
\hline $\mathrm{H}$ & -0.10477300 & -4.49642100 & 1.38457700 \\
\hline $\mathrm{C}$ & 0.53101400 & -6.49539400 & -0.47823000 \\
\hline $\mathrm{H}$ & 1.99063000 & -5.86358400 & 0.99121900 \\
\hline $\mathrm{H}$ & 2.18736000 & -5.10552100 & -0.59108100 \\
\hline $\mathrm{C}$ & 1.30010800 & -7.72521300 & -0.97217400 \\
\hline $\mathrm{H}$ & -0.03254100 & -6.05384100 & -1.32168600 \\
\hline
\end{tabular}




\begin{tabular}{|c|c|c|c|}
\hline $\mathrm{H}$ & -0.22960300 & -6.81237400 & 0.26028800 \\
\hline $\mathrm{C}$ & 0.39792600 & -8.79277000 & -1.59540500 \\
\hline $\mathrm{H}$ & 1.86400800 & -8.16450300 & -0.12843700 \\
\hline $\mathrm{H}$ & 2.05959900 & -7.40662200 & -1.70982100 \\
\hline $\mathrm{H}$ & -0.35024700 & -9.15760400 & -0.87087900 \\
\hline $\mathrm{H}$ & 0.97946300 & -9.66286000 & -1.94043300 \\
\hline $\mathrm{H}$ & -0.15274200 & -8.39378400 & -2.46440200 \\
\hline S & 2.21509600 & 1.73265600 & -1.37961200 \\
\hline $\mathrm{C}$ & 3.40264400 & 0.78434100 & -0.45798800 \\
\hline $\mathrm{C}$ & 2.80586700 & -0.17222800 & 0.33464100 \\
\hline $\mathrm{H}$ & 3.37611900 & -0.81387000 & 1.00352800 \\
\hline $\mathrm{C}$ & 4.80550800 & 1.04447900 & -0.58117800 \\
\hline S & 6.00545900 & -0.13657700 & 0.14003400 \\
\hline $\mathrm{O}$ & 5.91447600 & -0.15771100 & 1.62565700 \\
\hline $\mathrm{O}$ & 5.96542200 & -1.42368600 & -0.59992100 \\
\hline $\mathrm{C}$ & 5.51350300 & 2.02618000 & -1.20028700 \\
\hline $\mathrm{C}$ & 6.96072900 & 1.93089900 & -1.10589400 \\
\hline $\mathrm{H}$ & 5.03607200 & 2.85240300 & -1.72944100 \\
\hline $\mathrm{C}$ & 7.44265300 & 0.86596600 & -0.41001700 \\
\hline $\mathrm{H}$ & 7.60596000 & 2.68585400 & -1.55751300 \\
\hline $\mathrm{C}$ & 8.76119300 & 0.42054400 & -0.08486000 \\
\hline S & 10.19502900 & 1.20046900 & -0.76677900 \\
\hline $\mathrm{C}$ & 11.25726700 & 0.11681200 & 0.11351500 \\
\hline
\end{tabular}




$\begin{array}{llll}\mathrm{C} & 9.13313800 & -0.61048000 & 0.76114200 \\ \mathrm{C} & 10.53606700 & -0.78410600 & 0.87392600 \\ \mathrm{H} & 8.40442900 & -1.21349700 & 1.30187100 \\ \mathrm{H} & 10.99251200 & -1.54805300 & 1.50013900 \\ \mathrm{~S} & 12.99740700 & 0.31435200 & -0.08692400 \\ \mathrm{C} & 13.63050300 & -1.02981200 & 0.97341800 \\ \mathrm{H} & 14.72485500 & -0.97268300 & 0.89308000 \\ \mathrm{H} & 13.28738600 & -2.00948600 & 0.61105900 \\ \mathrm{H} & 13.33243000 & -0.87730400 & 2.02065800\end{array}$

Neurosporene//DFT(B3LYP)/cc-pVDZ

$\begin{array}{lllll}\text { C } & -17.71340100 & 3.04807300 & -0.96886700\end{array}$

$\begin{array}{lllll}\text { C } & -16.88647900 & 1.79955100 & -1.16125500\end{array}$

$\mathrm{H} \quad \begin{array}{llll}\mathrm{H} & -18.37343700 & 3.22504700 & -1.83780600\end{array}$

$\begin{array}{lllll}\mathrm{H} & -17.08588800 & 3.94086000 & -0.82866900\end{array}$

$\begin{array}{llll}\mathrm{H} & -18.37769600 & 2.95305400 & -0.09046400\end{array}$

$\begin{array}{lllll}\text { C } & -17.69613200 & 0.54221500 & -1.36065700\end{array}$

$\begin{array}{lllll}\mathrm{H} & -18.34415000 & 0.63390100 & -2.25119600\end{array}$

$\begin{array}{lllll}\mathrm{H} & -18.37316300 & 0.37719000 & -0.50309400\end{array}$

$\mathrm{H} \quad-17.08171100 \quad-0.35958900 \quad-1.48295700$

$\begin{array}{lllll}\text { C } & -15.54237400 & 1.85548400 & -1.15345400\end{array}$

$\begin{array}{lllll}\text { C } & -14.55266300 & 0.73380700 & -1.31655400\end{array}$

$\begin{array}{llll}\mathrm{H} & -15.08771100 & 2.84224500 & -0.99202800\end{array}$

$\begin{array}{lllll}\text { C } & -13.68776900 & 0.52456700 & -0.04841000\end{array}$ 


\begin{tabular}{|c|c|c|c|}
\hline $\mathrm{H}$ & -13.87240000 & 0.96952000 & -2.15645000 \\
\hline $\mathrm{H}$ & -15.06062900 & -0.20513800 & -1.58348100 \\
\hline $\mathrm{C}$ & -12.59701700 & -0.51745900 & -0.20056500 \\
\hline $\mathrm{H}$ & -14.35975900 & 0.23830800 & 0.78261300 \\
\hline $\mathrm{H}$ & -13.23402400 & 1.48919900 & 0.23438000 \\
\hline $\mathrm{C}$ & -13.07590200 & -1.93871600 & -0.37732500 \\
\hline $\mathrm{H}$ & -13.79814800 & -2.20351400 & 0.41522500 \\
\hline $\mathrm{H}$ & -12.26194400 & -2.67530200 & -0.35016600 \\
\hline $\mathrm{H}$ & -13.60658400 & -2.06758000 & -1.33707900 \\
\hline $\mathrm{C}$ & -11.30254200 & -0.14912900 & -0.17822300 \\
\hline $\mathrm{C}$ & -10.07492900 & -1.00962000 & -0.30786300 \\
\hline $\mathrm{H}$ & -11.09262200 & 0.91877800 & -0.02950100 \\
\hline $\mathrm{C}$ & -9.20053100 & -0.97985300 & 0.97299300 \\
\hline $\mathrm{H}$ & -9.45926600 & -0.63854100 & -1.14839800 \\
\hline $\mathrm{H}$ & -10.34231000 & -2.04777700 & -0.55538100 \\
\hline $\mathrm{C}$ & -7.89969400 & -1.74173300 & 0.85658300 \\
\hline $\mathrm{H}$ & -9.80011400 & -1.39569100 & 1.80487700 \\
\hline $\mathrm{H}$ & -8.98931500 & 0.07076200 & 1.23300200 \\
\hline $\mathrm{C}$ & -8.02205600 & -3.23824000 & 0.72625000 \\
\hline $\mathrm{H}$ & -8.67874200 & -3.64208300 & 1.51739400 \\
\hline $\mathrm{H}$ & -7.05817900 & -3.75971000 & 0.79110600 \\
\hline $\mathrm{H}$ & -8.48673900 & -3.52002800 & -0.23561400 \\
\hline $\mathrm{C}$ & -6.71654200 & -1.07460200 & 0.86769200 \\
\hline
\end{tabular}




\begin{tabular}{|c|c|c|c|}
\hline $\mathrm{C}$ & -5.38454500 & -1.62745700 & 0.75631200 \\
\hline $\mathrm{H}$ & -6.76050100 & 0.01627600 & 0.97518000 \\
\hline $\mathrm{C}$ & -4.25601300 & -0.86429400 & 0.78045500 \\
\hline $\mathrm{H}$ & -5.28810200 & -2.71027800 & 0.64567100 \\
\hline $\mathrm{C}$ & -2.89050100 & -1.33698300 & 0.67771400 \\
\hline $\mathrm{H}$ & -4.37575100 & 0.22061100 & 0.88800600 \\
\hline $\mathrm{C}$ & -2.65303300 & -2.81948900 & 0.52977900 \\
\hline $\mathrm{H}$ & -3.07853400 & -3.37209800 & 1.38472600 \\
\hline $\mathrm{H}$ & -1.58865400 & -3.07542600 & 0.46380500 \\
\hline $\mathrm{H}$ & -3.14788400 & -3.20734800 & -0.37693300 \\
\hline $\mathrm{C}$ & -1.87386200 & -0.41271400 & 0.72073600 \\
\hline $\mathrm{C}$ & -0.46326400 & -0.64540100 & 0.64162700 \\
\hline $\mathrm{H}$ & -2.17154800 & 0.63721500 & 0.83004200 \\
\hline $\mathrm{C}$ & 0.47544000 & 0.35157100 & 0.69435000 \\
\hline $\mathrm{H}$ & -0.10612300 & -1.67452400 & 0.53361400 \\
\hline $\mathrm{C}$ & 1.88490600 & 0.11790000 & 0.61754100 \\
\hline $\mathrm{H}$ & 0.11855800 & 1.38080300 & 0.80200000 \\
\hline $\mathrm{C}$ & 2.90411500 & 1.04222600 & 0.66079100 \\
\hline $\mathrm{H}$ & 2.18238500 & -0.93243800 & 0.51174700 \\
\hline $\mathrm{C}$ & 2.66605700 & 2.52587300 & 0.80231100 \\
\hline $\mathrm{H}$ & 1.60133100 & 2.78134600 & 0.86413600 \\
\hline $\mathrm{H}$ & 3.09294500 & 3.07530800 & -0.05389100 \\
\hline $\mathrm{H}$ & 3.15772800 & 2.91780300 & 1.70887800 \\
\hline
\end{tabular}




\begin{tabular}{|c|c|c|c|}
\hline C & 4.26480600 & 0.56773400 & 0.56680200 \\
\hline $\mathrm{C}$ & 5.39917800 & 1.33184100 & 0.59282800 \\
\hline $\mathrm{H}$ & 4.38504900 & -0.51757300 & 0.46541800 \\
\hline $\mathrm{C}$ & 6.71912100 & 0.77806800 & 0.49505500 \\
\hline $\mathrm{H}$ & 5.30120700 & 2.41528000 & 0.69320400 \\
\hline $\mathrm{C}$ & 7.92130100 & 1.44220600 & 0.51096900 \\
\hline $\mathrm{H}$ & 6.76430700 & -0.31329100 & 0.39613900 \\
\hline $\mathrm{C}$ & 8.03586200 & 2.94112800 & 0.64004600 \\
\hline $\mathrm{H}$ & 7.06075300 & 3.43660400 & 0.72158800 \\
\hline $\mathrm{H}$ & 8.55864500 & 3.37143300 & -0.23119400 \\
\hline $\mathrm{H}$ & 8.62635600 & 3.21441100 & 1.53116400 \\
\hline $\mathrm{C}$ & 9.13978800 & 0.66523700 & 0.39922200 \\
\hline $\mathrm{C}$ & 10.41405900 & 1.14672400 & 0.39624600 \\
\hline $\mathrm{H}$ & 9.00371600 & -0.41928400 & 0.30825400 \\
\hline $\mathrm{C}$ & 11.58068400 & 0.29870300 & 0.28136600 \\
\hline $\mathrm{H}$ & 10.57219600 & 2.22457700 & 0.48188700 \\
\hline $\mathrm{C}$ & 12.88614700 & 0.67312200 & 0.27052700 \\
\hline $\mathrm{H}$ & 11.36941800 & -0.77432600 & 0.19457500 \\
\hline $\mathrm{C}$ & 13.35419000 & 2.10095200 & 0.38891800 \\
\hline $\mathrm{H}$ & 13.81842500 & 2.45009300 & -0.55083500 \\
\hline $\mathrm{H}$ & 14.13104100 & 2.18911700 & 1.16931600 \\
\hline $\mathrm{H}$ & 12.54726100 & 2.80259600 & 0.63753100 \\
\hline C & 13.97251500 & -0.36878700 & 0.12665200 \\
\hline
\end{tabular}




\begin{tabular}{|c|c|c|c|}
\hline $\mathrm{C}$ & 14.75890900 & -0.29108700 & -1.20827000 \\
\hline $\mathrm{H}$ & 13.53118200 & -1.37509900 & 0.21954900 \\
\hline $\mathrm{H}$ & 14.69780100 & -0.26515000 & 0.95595200 \\
\hline $\mathrm{C}$ & 15.75324600 & -1.41147200 & -1.34933200 \\
\hline $\mathrm{H}$ & 15.25185700 & 0.68882900 & -1.29487900 \\
\hline $\mathrm{H}$ & 14.02818700 & -0.34701300 & -2.03654900 \\
\hline $\mathrm{C}$ & 17.09506400 & -1.34115500 & -1.41631000 \\
\hline $\mathrm{H}$ & 15.30602200 & -2.41398100 & -1.38524100 \\
\hline $\mathrm{C}$ & 17.89474300 & -0.06247800 & -1.37674300 \\
\hline $\mathrm{H}$ & 18.49269300 & 0.05095200 & -2.29919900 \\
\hline $\mathrm{H}$ & 18.61808300 & -0.08365300 & -0.54171500 \\
\hline $\mathrm{H}$ & 17.27636200 & 0.83782900 & -1.26373300 \\
\hline $\mathrm{C}$ & 17.92896500 & -2.59279700 & -1.54861700 \\
\hline $\mathrm{H}$ & 18.64049600 & -2.68651200 & -0.70791100 \\
\hline $\mathrm{H}$ & 18.54004200 & -2.56713200 & -2.46942100 \\
\hline $\mathrm{H}$ & 17.30851100 & -3.50097300 & -1.57509300 \\
\hline
\end{tabular}




\section{References}

(1) Feng, X.; Luzanov, A. V.; Krylov, A. I. Fission of Entangled Spins: An Electronic Structure Perspective. J. Phys. Chem. Lett. 2013, 4 (22), 3845-3852. https://doi.org/10.1021/jz402122m.

(2) Scholes, G. D. Correlated Pair States Formed by Singlet Fission and Exciton-Exciton Annihilation. J. Phys. Chem. A 2015, 119, 12699-12705. https://doi.org/10.1021/acs.jpca.5b09725.

(3) Berkelbach, T. C.; Hybertsen, M. S.; Reichman, D. R. Microscopic Theory of Singlet Exciton Fission. II. Application to Pentacene Dimers and the Role of Superexchange. The Journal of Chemical Physics 2013, 138 (11), 114103. https://doi.org/10.1063/1.4794427.

(4) Bayliss, S. L.; Weiss, L. R.; Mitioglu, A.; Galkowski, K.; Yang, Z.; Yunusova, K.; Surrente, A.; Thorley, K. J.; Behrends, J.; Bittl, R.; Anthony, J. E.; Rao, A.; Friend, R. H.; Plochocka, P.; Christianen, P. C. M.; Greenham, N. C.; Chepelianskii, A. D. Site-Selective Measurement of Coupled Spin Pairs in an Organic Semiconductor. PNAS 2018, 115 (20), 5077-5082. https://doi.org/10.1073/pnas.1718868115. 